\title{
Human Inborn Errors of Immunity: 2019 Update of the IUIS Phenotypical Classification
}

\author{
Aziz Bousfiha $^{1,2} \cdot$ Leila Jeddane $^{3} \cdot$ Capucine Picard $^{4,5} \cdot$ Waleed Al-Herz $^{6}$ • Fatima Ailal $^{1} \cdot$ Talal Chatila $^{7}$. \\ Charlotte Cunningham-Rundles $^{8}$ - Amos Etzioni ${ }^{9}$. Jose Luis Franco ${ }^{10}$. Steven M Holland ${ }^{11}$. Christoph Klein ${ }^{12}$. \\ Tomohiro Morio ${ }^{13}$. Hans D. Ochs ${ }^{14}$. Eric Oksenhendler ${ }^{15}$. Jennifer Puck ${ }^{16}$. Troy R. Torgerson ${ }^{14}$. \\ Jean-Laurent Casanova ${ }^{17,18,19,20} \cdot$ Kathleen E. Sullivan ${ }^{21} \cdot$ Stuart G. Tangye ${ }^{22,23}$
}

Received: 12 December 2019 / Accepted: 22 January 2020 / Published online: 11 February 2020

(C) The Author(s) 2020

\begin{abstract}
Since 2013, the International Union of Immunological Societies (IUIS) expert committee (EC) on Inborn Errors of Immunity (IEI) has published an updated phenotypic classification of IEI, which accompanies and complements their genotypic classification into ten tables. This phenotypic classification is user-friendly and serves as a resource for clinicians at the bedside. There are now 430 single-gene IEI underlying phenotypes as diverse as infection, malignancy, allergy, autoimmunity, and autoinflammation. We herein report the 2019 phenotypic classification, including the 65 new conditions. The diagnostic algorithms are based on clinical and laboratory phenotypes for each of the ten broad categories of IEI.
\end{abstract}

Keywords IUIS · primary immune deficiency $\cdot$ inborn errors of immunity $\cdot$ immune dysregulation $\cdot$ autoinflammatory disorders · classification

\section{Introduction}

Human inborn errors of immunity (IEI) are caused by monogenic germline mutations resulting in loss or gain of function of the encoded protein. They can be dominant or recessive, autosomal or X-linked, and with complete or incomplete penetrance. They manifest as increased susceptibility to a broad or narrow spectrum of infectious diseases, as well as a growing diversity of autoimmune, autoinflammatory, allergic, and/or malignant phenotypes. They now comprise 406 distinct disorders with 430 different gene defects listed in the 2019 International Union of Immunological Societies (IUIS) classical classification [1]. If most IEI are individually rare, they are collectively more common than generally thought [2].

The (IUIS) expert committee on IEI proposes every other year a genotypic classification of all these disorders [1], which facilitates both research on, and diagnosis of, these conditions worldwide. This classification is organized in ten tables, each

Aziz Bousfiha

profbousfiha@gmail.com

Extended author information available on the last page of the article of which groups IEI sharing a given pathogenesis. However, with the growing number of IEI included in this catalog, these tables are not always easy to use at the bedside. We thus reported from 2013 onward a more user-friendly classification adapted for the clinician, based on the clinical and laboratory features observed in these patients. This phenotypic classification proved to be as popular as the genotypic classification (15 k vs $12 \mathrm{k}$ downloads on publisher site) [3] and has been adapted in a smartphone application [4].

Here, we present an update of the phenotypic classification of IEI, based on the 2019 IEI classical classification [1]. This tree-based decision-making process is aimed to physicians, regardless of their familiarity with IEI. It aims at helping them to reach a diagnosis based on simple clinical and biological phenotypes.

\section{Methodology}

We included in our figures all disorders indexed in the 2019 update of the IUIS IEI classification [1]. A phenotypic algorithm was assigned to each of the ten main groups of the classification and the same color was used for each group of similar conditions. Given the high 
I. Immunodeficiencies affecting cellular and humoral immunity.

(a) Severe combined immunodeficiencies SCID, defined by CD3 T cell lymphopenia*.

\begin{tabular}{|c|c|c|}
\hline \multicolumn{3}{|c|}{ CD19 NL : SCID T- B+ } \\
\hline SCID T-B+NK- & \multicolumn{2}{|c|}{ SCID T-B+NK+ } \\
\hline XI & $\begin{array}{l}\text { IL7R } \alpha . \\
\text { IL7R }\end{array}$ & $\begin{array}{c}\text { Coronin-1A def *. } \\
\text { CORO1A }\end{array}$ \\
\hline & 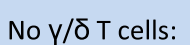 & Detectable \\
\hline $\begin{array}{l}\text { CD } 132 \text { def } \\
\gamma c \text { deficiency }\end{array}$ & $\begin{array}{l}C D 3 \delta^{*} \cdot C D 3 D \\
C D 3 \varepsilon^{*} \cdot C D 3 E \\
C D 3 \zeta^{* *} . C D 3 Z\end{array}$ & $\begin{array}{l}\text { Winged helix def*. } \\
\text { FOXN1. }\end{array}$ \\
\hline IL2RG & & Severe infections: \\
\hline AR, & $\begin{array}{l}\text { CD45* } \\
\text { PTPRC }\end{array}$ & $\begin{array}{l}\text { abnormal thymic } \\
\text { epithelium; }\end{array}$ \\
\hline CD 132+ & LAT def*. LAT. & alopecia, nail \\
\hline JAK-3 def & $\begin{array}{l}\text { Typical SCID or } \\
\text { CID with } \\
\text { adenopathy, } \\
\text { splenomegaly, } \\
\text { autoimmunity. } \\
\text { High Ig. }\end{array}$ & $\begin{array}{l}\text { dystrophy, neural } \\
\text { tube defect. Ig: } \\
\text { decreased .Tc: } \\
\text { Very low. }\end{array}$ \\
\hline
\end{tabular}

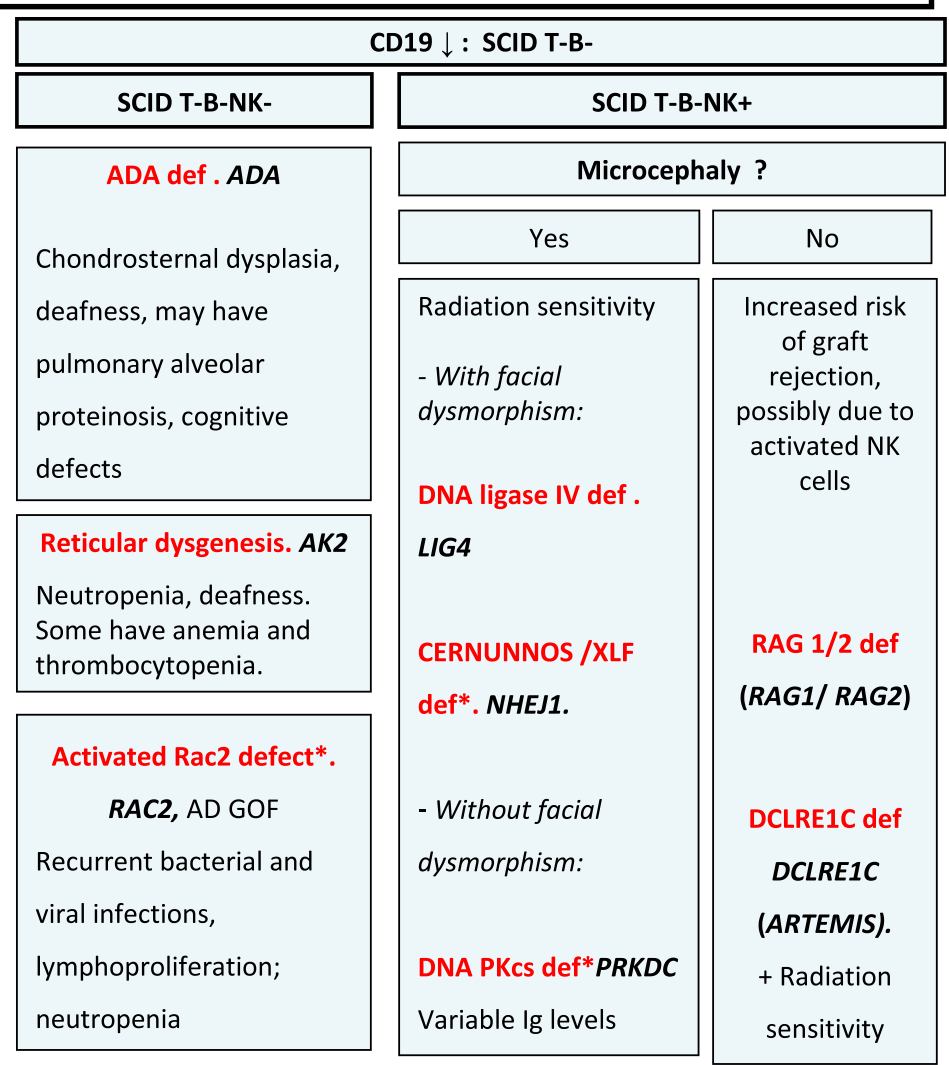

Fig. 1 Immunodeficiencies affecting cellular and humoral immunity. a Severe combined immunodeficiencies defined by $\mathrm{T}$ cell lymphopenia. $\mathbf{b}$ Combined immunodeficiencies. * T cell lymphopenia in SCID is defined by $\mathrm{CD} 3+\mathrm{T}$ cells $<300 / \mu \mathrm{L}$. AD autosomal dominant transmission, ADA adenosine deaminase, Adp adenopathies, Ag antigen, AR autosomal recessive transmission, $\beta 2 \mathrm{~m}$ bêta- 2 microglobulin, $\mathrm{Bc} B$ cells, $\mathrm{CBC}$ complete blood count, CD cluster of differentiation, CVID common

number of diseases, several categories have been split since last update [3] in two sub-figures to be more informative.

Disease names are presented in red and genes in bold italic. An asterisk is added to highlight extremely rare disorders (less than 10 reported cases to date). However, the reader should keep in mind that some genes have been very recently described and that true prevalence is unknown. A double asterisk is added when only one case or one kindred has been reported to date. In these cases, it is difficult to confirm than observed phenotype would be reproducible in other patients carrying the same defect, or if it is an exception.

\section{Results}

Algorithms for the 2019 update of IUIS phenotypical classification are presented in Figs. 1, 2, 3, 4, 5, 6, 7, 8, 9, and 10. variable immunodeficiency, def deficiency, EBV Epstein-Barr virus, Eo eosinophilia, GOF gain-of-function mutation, HHV8 human herpes virus 8, HIGM hyper IgM syndrome, HPV human papillomavirus, HSM hepatosplenomegaly, Ig immunoglobulins, MHC major histocompatibility complex, Nl normal, NK natural killer, SCID severe combined immunodeficiency, Tc T cells, TCR T cell receptor, Treg regulatory $\mathrm{T}$ cells, $\mathrm{XL}$ X-linked transmission

\section{Discussion}

These algorithms are aimed to guide clinicians to diagnose patients presenting typical phenotype. However, readers should be aware of the limitations of such a work.

More and more reports show a spectrum of atypical presentations related to hypomorphic mutations of those genes. Omenn syndrome (OMIM \#603554) is a good example of such an atypical presentation, as well as "leaky SCID" and RAG deficiency spectrum [5].

Moreover, readers should be extremely cautious with descriptions of disease when only one patient or kindred have been reported. We are aware that these reports may not reflect the typical phenotype of such defects, but the exception; however, we thought that it was needed to be mentioned in these classifications. 


\begin{tabular}{|c|c|c|c|}
\hline & ombined Immu & $\begin{array}{l}\text { nunodeficienci } \\
\text { ficiencies Gene }\end{array}$ & $\begin{array}{l}\text { ecting cellular } \\
\text { ess Profound }\end{array}$ \\
\hline \multicolumn{2}{|c|}{$\begin{array}{ll}\text { Low CD4: } & \text { Low CD8 } \\
\end{array}$} & Low Bc: & Ig : often NL \\
\hline \multicolumn{2}{|c|}{ MHCII Expression ? } & \multirow{2}{*}{$\begin{array}{l}\text { Omenn sd (hypomorphic } \\
\text { mutations). Erythroderma, } \\
\text { Alopecia, Adp, HSM, Eo } \uparrow, \text { IgE } \uparrow\end{array}$} & \multirow{2}{*}{$\begin{array}{l}\text { CD3y def*. CD3G TCR low. } \\
\text { Autoimmunity }\end{array}$} \\
\hline Absent & Present & & \\
\hline \multirow{5}{*}{$\begin{array}{l}\text { MHC-II def } \\
\text { RFXANK,CIIT } \\
\text { A, RFX5, } \\
\text { RFXAP } \\
\text { AR, Failure to } \\
\text { thrive, } \\
\text { respiratory } \\
\text { and } \\
\text { gastrointesti } \\
\text { nal } \\
\text { infections, } \\
\text { liver/biliary } \\
\text { tract disease }\end{array}$} & $\begin{array}{l}\text { LCK def. LCK. AR, Immune } \\
\text { dysregulation, auto-immunity. } \\
\text { Low Treg, restricted T cell } \\
\text { repertoire, poor TCR } \\
\text { signaling. } \uparrow \text { IgM. }\end{array}$ & \multirow[t]{2}{*}{$\begin{array}{l}\text { DOCK8 def. DOCK8.Severe } \\
\text { Eczema. Cutaneous viral and } \\
\text { staphylococcal infections; severe } \\
\text { atopy; cancer, diathesis. High IgE, } \\
\text { Low IgM, eosinophilia. } \downarrow \text { NK with } \\
\text { poor function. } \uparrow \mathrm{Bc}, \downarrow \text { memory Bc } \\
\text { Poor peripheral Bc tolerance. } \uparrow \\
\text { exhausted CD8+ TEM cells } \\
\end{array}$} & $\begin{array}{l}\text { RHOH def**. RHOH. HPV } \\
\text { infection, lung granulomas, } \\
\text { molluscum contagiosum, } \\
\text { lymphoma. Low naïve T } \\
\text { cells, restricted repertoire, } \\
\text { poor proliferation to CD3. }\end{array}$ \\
\hline & Polymerase $\delta$ def*. AR. & & TCR $\alpha$ def* .TRAC. \\
\hline & $\begin{array}{l}\text { POLD1 or POLD2. } \\
\text { Recurrent respiratory tract } \\
\text { infections, skin infections, warts } \\
\text { and molluscum, short stature, } \\
\text { intellectual disability. Low Bc, } \\
\text { Low Ig. }\end{array}$ & \multirow{4}{*}{$\begin{array}{l}\text { STK4 def. STK4. Intermittent } \\
\text { neutropenia, bacterial, viral (HPV, } \\
\text { EBV, molluscum), candidal } \\
\text { infections, lymphoproliferation, } \\
\text { autoimmune cytopenias, } \\
\text { lymphoma, congenital heart } \\
\text { disease. } \downarrow: \text { : CD4 Tc, naive Tc, } \uparrow \\
\text { TEM and TEMRA cells, poor } \\
\text { proliferation. } \downarrow \text { : memorr Bc, IgM } \\
\text { \& Ab responses. } \uparrow \text { IgG, IgA, IgE. }\end{array}$} & $\begin{array}{l}\text { Recurrent viral, bacterial, } \\
\text { fungal infections; diarrhea; } \\
\text { immune dysregulation and } \\
\text { autoimmunity. Absent } \\
\text { TCR } \beta \text { except for a minor } \\
\text { CD3-dim TCR } \alpha \beta \text { population; }\end{array}$ \\
\hline & $A D:$ UNC119 def & & poor proliferation. \\
\hline & UNC119 & & OX40 def**. ox40. \\
\hline \multirow{2}{*}{\multicolumn{2}{|c|}{$\begin{array}{l}\text { CD8 def *. CD8A } \\
\text { Recurrent infections .Maybe asymptomatic.CD8 Absent. }\end{array}$}} & & $\begin{array}{l}\text { Kaposi's sarcoma, impaired } \\
\text { immunity to HHV8. Low }\end{array}$ \\
\hline & & IL21 def.** IL21. Severe early & memory Bc. Tc : low Ag \\
\hline \multirow{2}{*}{\multicolumn{2}{|c|}{$\begin{array}{l}\text { NI MHC -I on lymphocytes. } \\
\text { ZAP-70 def. ZAP7O May have immune dysregulation, } \\
\text { autoimmunity. NI Ig. CD4: Low fonction } \\
\text { Combined hypomorphic and activating } \\
\text { mutations: Severe autoimmunity . NI or decreased CD4 } \\
\text { and Bc. NI IgA, low IgM, IgG NI or low. }\end{array}$}} & $\begin{array}{l}\text { function. Hypogamma- } \\
\text { globulinemia, poor specific } \\
\text { antibody responses; } \uparrow \text { IgE }\end{array}$ & FCHO1 def*. FCHO1 \\
\hline & & $\begin{array}{l}\text { NIK def**. MAP3K14. } \\
\text { Bacterial, viral and } \\
\text { Cryptosporidium infections. } \downarrow \text { : }\end{array}$ & $\begin{array}{l}\text { Lymphoproliferation, } \\
\text { failure to thrive. . Tc: Low. } \\
\text { Bc \& Ig : NI Increased }\end{array}$ \\
\hline \multirow{2}{*}{\multicolumn{2}{|c|}{$\begin{array}{l}\text { Absent MHC -I on lymphocytes. } \\
\text { MHC-I def. } \\
\text { TAP2, TAP1 or TAPBP : Vasculitis, pyoderma } \\
\text { gangrenosum. NI Ig. } \\
\text { B2M *: Sinopulmonary infections, cutaneous } \\
\text { granulomas. NI Ig. Hypoprotidemia. Absent } \beta 2 \mathrm{~m} \\
\text { associated proteins MHC-I, CD1a, CD1b, CD1c. }\end{array}$}} & $\begin{array}{l}\text { NK, Ig levels \& switched m } \\
\text { Bc. Tc :Ag poor proliferatic }\end{array}$ & $\begin{array}{l}\text { death, defective clathrin- } \\
\text { mediated endocytosis }\end{array}$ \\
\hline & & $\begin{array}{l}\text { Moesin def.*MSN. XL, } \\
\text { Recurrent infections with } \\
\text { bacteria, varicella; neutropenia. } \\
\downarrow \text { Ig over time. Tc: defective } \\
\text { migration, proliferation. }\end{array}$ & $\begin{array}{l}\text { RelA haploinsufficiency**. } \\
\text { RELA, AD. } \\
\text { Chronic mucocutaneous } \\
\text { ulceration. Impaired NFkB }\end{array}$ \\
\hline \multicolumn{3}{|c|}{$\begin{array}{l}\text { C-REL def**. REL.: Recurrent infections with bacteria, mycobacteria, salmonella and opportunistic } \\
\text { organisms. Defective innate immunity. Low Ig. Tc: decreased memory CD4, poor proliferation. }\end{array}$} & of inflammatory cytokines \\
\hline \multicolumn{3}{|c|}{$\begin{array}{l}\text { ICOSL def**. ICOSL. Recurrent respiratory tract viral infections. hypogammaglobulinemia, and Low } \\
\text { TC, slowly progressive neutropenia }\end{array}$} & $\begin{array}{l}\text { ITK deficiency. ITK. } \\
\text { EBV associated Bc lympho- } \\
\text { proliferation, lymphoma, }\end{array}$ \\
\hline \multicolumn{3}{|c|}{$\begin{array}{l}\text { IKAROS def*. (CD154). AD DN, IKZF1. Opportunistic infections, including P.jirovecii, bacterial, } \\
\text { viral and other fungal infections. Increased risk fo T-ALL. Agammaglobulinemia, high recent thymic } \\
\text { emigrant/naive/ThO cells; low-absent memory T cells }\end{array}$} & $\begin{array}{l}\text { low IgG. Progressive CD4 T cell } \\
\text { lymphopenia; reduced T cell } \\
\text { activation }\end{array}$ \\
\hline
\end{tabular}

Fig. 1 (continued) 


\section{Ila. CID with associated or syndromic features}

\begin{tabular}{|c|c|}
\hline Congenital thrombocytopenia & DNA Repair Defects other than those listed \\
\hline \multicolumn{2}{|c|}{$\begin{array}{l}X L \text { : Wiskott Aldrich Sd or XL thrombocytopenia WAS (LOF). Recurrent bacterial and viral infections; bloody diarrhea; eczema; } \\
\text { lymphoma; autoimmune disease; IgA nephropathy; vasculitis. Small platelets; Decreased IgM. Low antibody to polysaccharides; } \\
\text { often increased IgA and IgE. NI Bc. Tc: Progressive decrease in numbers; Low Tc responses to anti-CD3. } \\
\text { Patients with XL-thrombocytopenia have later onset of complications and more favourable life expectancy but eventually } \\
\text { develop similar complications as observed in WAS }\end{array}$} \\
\hline $\begin{array}{l}\text { AR: WIP deficiency*. WIPF1, WAS protein absen } \\
\text { lymphocyte responses to anti-CD3. }\end{array}$ & ts; increased IgE. Bc : NI to low. Tc: Reduced; defective \\
\hline
\end{tabular}

Ataxia telangiectasia. ATM: Ataxia; telangiectasia; pulmonary infections; lymphoreticular and other malignancies; increased $\alpha$ fetoprotein; increased radiosensitivity, chromosomal instability and translocations. Often decreased IgA, IgE and IgG subclasses; increased IgM; antibodies variably decreased. Tc: Progressive decrease, abnormal prolif to Mitogens.

Nijmen Often decreased IgA, IgE and IgG subclasses;increased IgM; antibodies variably decreased. Bc: Variably reduced. Tc: progressive decrease.

Bloom sd. BLM.Short stature; bird like face; sun-sensitive erythema; marrow failure; leukemia; lymphoma; chromosomal instability. Low Ig.

PMS2 def. PMS2. Café-au-lait spots ; lymphoma, colorectal carcinoma, brain tumors. HIGM and abnormal antibody responses. Reduced $\mathrm{Bc}$, switched and non-switched.

Immunodeficiency with centromeric instabiltiy and facial anomalies: ICF1. DNMT3B; ICF2:ZBTB24; ICF3:CDCA7; ICF4:HELLS.Facial dysmorphism; macroglossia; bacterial/opportunistic infections; malabsorption; malignancies. Cytopenias; multiradial configurations of chromosomes 1,9,16; no DNA breaks. Ig: Hypogammaglobulinemia; Tc and Bc: decreased or NI.

MCM4 def. MCM4. Viral infections:EBV,HSV,VZV.short stature.Bc lymphoma; Adrenal failure; NKc low number and function.

RNF168 def* (RIDDLE sd). RNF168. Short stature; mild defect of motor control to ataxia; normal intelligence to learning difficulties; mild facial dysmorphism to microcephaly; increased radiosensitivity. Low IgG or IgA.

POLE1 (Polymerase $\varepsilon$ subunit 1) deficiency (FILS syndrome). POLE1. Recurrent respiratory infections; meningitis; facial dysmorphism, livido, short stature. Low IgM, lack of antibody to PPS. Low memory Bc. Decreased Tc proliferation.

POLE2 (Polymerase $\varepsilon$ subunit 2) deficiency**. POLE2. Recurrent infection, disseminated BCG infections, autoimmunity (type 1 diabetes, hypothyroidism), facial dysmorphism; Low Ig; Very low Bc. Lymphopenia, lack of TRECS, absent proliferation of antigens.

NSMCE3 deficiency*. NSMCE3. Severe lung disease (possibly viral); thymic hypoplasia, Chromosomal breakage; radiation sensitivity. Ig: Decreased Ab responses to PPS, normal IgG, IgA, normal to elevated IgM. Tc : Low, poor responses to mitogens and antigens.

Ligase I deficiency *. LIGI Recurrent bacterial and viral infections; growth retardation; sun sensitivity; lymphoma; radiation sensitivity. Macrocytic red blood cells. Hypogammaglobulinemia. Reduced Ab response. Lymphopenia, increased $\gamma \delta \mathrm{TC}$, decreased mitogen response.

GINS1 def*. GINS1. IUGR. Neutropenia, NK cells very low. Tc and Bc: low or normal. High IgA, Low IgG and IgM.

BMFS2 (Hebo def). ERCC6L2, AR. Facial dysmorphism; microcephaly, learning difficulties. Bone marrow failure.

Fig. 2 a, b CID with associated or syndromic features. Ab antibody, AD autosomal dominant transmission, AD DN autosomal dominant transmission with dominant negative effect, ANA anti-nuclear antibodies, ANCA anti-neutrophil cytoplasm antibodies, AR autosomal recessive transmission, $\mathrm{Bc}$ B cells, BCG bacillus Calmette-Guerin, BCR $B$ cell receptor, $C D$ cluster of differentiation, CID combined immunodeficiency of $\mathrm{T}$ and $\mathrm{B}$ cells, CMV cytomegalovirus, CNS central nervous system, def deficiency, DNA deoxyribonucleic acid, EBV Epstein-Barr virus, EDA anhidrotic ectodermal dysplasia, GOF gain-of-function, HIES hyper IgE syndrome, FILS facial dysmorphism, immunodeficiency, livedo and short stature, ID immunodeficiency, Ig immunoglobulins, IL-6 interleukin-6, IUGR intrauterine growth retardation, LOF loss-of-function, MCC mucocutaneous candidiasis, N1 normal, NK natural killer, PHA phytohemagglutinin, PPS polysaccharides, SCID severe combined immunodeficiency, sd syndrome, Tc T cells, TCR T cell receptor, TREC $\mathrm{T}$ cell receptor excision circle, XL X-linked transmission 


\section{IIb. CID with associated or syndromic features}

\begin{tabular}{|c|}
\hline Hyper-IgE syndromes (HIES) \\
\hline $\begin{array}{l}\text { AD-HIES (Job sd ). STAT3, AD LOF. Distinctive facial features (broad } \\
\text { nasal bridge); bacterial infections (boils and pulmonary abscesses, } \\
\text { pneumatoceles) due to } S \text {. aureus, Aspergillus, Pneumocystis jirovecii; } \\
\text { eczema; mucocutaneous candidiasis; hyperextensible joints, } \\
\text { osteoporosis and bone fractures, scoliosis, retention of primary } \\
\text { teeth; aneurysm formation. IgE } \uparrow \uparrow ; \text { specific antibody production } \downarrow \text {. } \\
\text { Bc:Normal; reduced switched and non-switched memory Bc; BAFF } \\
\text { expression } \uparrow . \text { Tc:Nl overall; Th-17 \& T-follicular helper cells } \downarrow\end{array}$ \\
\hline $\begin{array}{l}\text { ZNF341 deficiency. ZNF341. AR. Phenocopy of AD-HIES: Mild facial } \\
\text { dysmorphism, early onset eczema, MCC, bacterial skin infections, } \\
\text { abscesses, recurrent bacterial respiratory infections (S. aureus), lung } \\
\text { abscesses and pneumatoceles, hyperextensible joints, bone fractures } \\
\text { and retention of primary teeth }\end{array}$ \\
\hline $\begin{array}{l}\text { Comel Netherton Sd; SPINK5; Congenital ichthyosis, bamboo } \\
\text { hair,atopic diathesis; } \uparrow \text { bacterial infections, failure to thrive. } \uparrow \text { IgE } \\
\text { and IgA; Other Ig: variably decreased. Bc: Switched and non-switched } \\
\text { Bc are } \downarrow \text {. }\end{array}$ \\
\hline $\begin{array}{l}\text { PGM3 deficiency. PGM3. Severe atopy; autoimmunity; skeletal } \\
\text { anomalies: short stature, brachydactyly, dysmorphic facial } \\
\text { features. Recurrent pneumonia, recurrent skin abscesses, bacterial } \\
\text { and viral infections; cognitive impairment; delayed CNS myelination } \\
\text { in some. Ig:NI or elevated. Elevated IgE; eosinophilia. Reduced B and } \\
\text { memory Bc. CD8 and CD4 Tc may be } \downarrow \text {. }\end{array}$ \\
\hline $\begin{array}{l}\text { CID with early-onset asthma, eczema and food allergies, } \\
\text { autoimmunity ID with atopic dermatitis (CADINS)*. CARD11. AD LOF. } \\
\text { Variable atopy, cutaneous viral infections, recurrent respiratory } \\
\text { infections, lymphoma. Eosinophilia, } \downarrow \text { Tc proliferation. NI to low Bc. }\end{array}$ \\
\hline 11 \\
\hline $\begin{array}{l}\text { ERBIN deficiency**. ERBB21P. Recurrent respiratory infections, } \\
\text { susceptibility to S. aureus, eczema, hyperextensible joints, scoliosis, } \\
\text { arterial dilatation in some. Moderately increased IgE; increased Treg. }\end{array}$ \\
\hline $\begin{array}{l}\text { IL6R deficiency*. IL6R. Recurrent pyogenic infections, cold } \\
\text { abscesses, high circulating IL-6 Levels. }\end{array}$ \\
\hline $\begin{array}{l}\text { IL6ST deficiency*. IL6ST. Bacterial infections, boiles, eczema, } \\
\text { pulmonary abscesses, pneumatoceles, bone fractures, scoliosis, } \\
\text { retention of primary teeth, craniosynostosis. } \downarrow \text { B-cell memory. }\end{array}$ \\
\hline $\begin{array}{l}\text { Loes-Dietz syndrome.TGFBR1, TGFBR2. Recurrent respiratory } \\
\text { infectons, eczema, food allergies, hyperextensible joints, scoliosis, } \\
\text { retention of primary teeths; aortic aneurisms. }\end{array}$ \\
\hline
\end{tabular}

Defects of Vitamin

B12 and Folate

Metabolism:

Megaloblastic anemia, Ig: decreased.

Transcobalamin 2

deficiency. TCN2.

pancytopenia, if untreated for

prolonged periods results in

intellectual disability.

Deficiency causing

hereditary folate

malabsorbtion. SLC46A1.

failure to thrive, if untreated

for prolonged periods results

in intellectual disability

Methylene-

tetrahydrofolate

dehydrogenase 1
deficiency MTHFD1.

deficiency MTHFD1.
Recurrent bacterial infection

Recurrent bacterial infection,
Pneumocystis jirovecii; failure

to thrive; neutropenia;

to thrive; neutropen
seizures, intellectual

disability; folate-responsive

$\downarrow \mathrm{BC}, \downarrow$ antibody responses

to conjugated
antigens.

\section{Anhidrotic \\ Ectodermodysplasia \\ with ID}

Anhidrotic ectodermal

Ansidrotic ectodermal

dysplasia, various infections
(bacteria, mycobacteria, viruses and

fungi), colitis, variable defects of

skin, hair and teeth.

NEMO deficiency. IKBKG (NEMO).

$\mathrm{XL}$, monocyte dysfunction. Ig

decreased, some with elevated IgA,

IgM, poor specific antibody

responses, absent antibody to
polysaccharide antigens. Bc: $\mathrm{Nl}$, Low

polysaccharide antigens. $\mathrm{Bc}$ : $\mathrm{Nl}, \mathrm{LOW}$
memory and isotype switched $\mathrm{BC}$.

TC: NI/decreased, TCR activation

impaired.

EDA-ID due to IKBA GOF mutation.

NFKBIA (IKBA). AD TC and

monocyte dysfunction Decreased

$\lg G$ and IgA, elevated IgM, poor

specific antibody responses, absen

antibody to polysaccharide

antigens. Normal Bc numbers,

impaired BCR activation, low

mpaired.

EDA-ID due to IKBK GOF mutation *

poor function. Low Is. memory and isotype switched $B C$

Normal total Tc, TCR activation

\begin{tabular}{|l|}
\hline Others \\
\hline Purine nucleoside phosphorylase deficiency. PNP. Autoimmune \\
haemolytic anemia, neurological impairment. Hypouricemia. Ig : NI/Low. \\
Bc: NI. Tc: Progressive decrease \\
\hline \hline Calcium Channel Defects. Autoimmunity, EDA, non-progressive myopathy. \\
Ig and BC: NI. Tc: Normal, defective TCR mediated activation. ORAl-1 \\
deficiency*. ORA11 . STIM1 deficiency*. STIM1 \\
\hline
\end{tabular}
deficiency*. ORAI1 . STIM1 deficiency*. STIM1

ID with multiple intestinal atresias. TTC7A . Bacterial (sepsis), fungal, viral infections, multiple intestinal atresias, often with intrauterine

polyhydramnios and early demise, some with SCID phenotype. Markedly decreased IgG, IgM, IgA. Bc:NI/low.Tc: Variable/absent, low TRECs (may present with SCID at birth)

Hepatic veno-occlusive disease with immunodeficiency (VODI). SP110. Hepatic veno-occlusive disease, Pneumocystis jirovecii pneumonia, CMV, candida, thrombocytopenia, hepatosplenomegaly, cerebrospinal leukodystrophy. Decreased IgG, IgA, IgM, absent germinal centers and tissue plasma cells. Decreased memory Bc. Decreased memory Tc.

STATSb deficiency. STATSB. AR. Growth-hormone insensitive dwarfism, dysmorphic features, eczema, lymphocytic interstitial pneumonitis, autoimmunity. Hypergammaglobulinemia, High IgE. $A D D N$ : Growth failure and eczema only. High IgE.

BCL11B deficiency. BCL11B. AD. Congenital abnormalities: neonatal teeth, dysmorphic facies; absent corpus callosum; neurocognitive deficits. Tc : Low, poor proliferation.

Hennekam-lymphangiectasia-lymphedema syndrome*. CCBE1, FAT4. 作 dysmorphic features. Ig: decreased. Bc and Tc: Variable.

Bacterial infections, autoinflammation amylopectinosis $B C$ : Nldecreased memory $B$.

Bacterial infections, autoinflammation, amylopectinosis.BC: NI, decreased memory BC.
HOIL1 deficiency. RBCK1. Poor Ab responses to polysaccharides. HOIP deficiency*. RNF31. Lymphangiectasia. Ig: decreased.

Vici syndrome. EPG5. Agenesis of the corpus callosum, cataracts, cardiomyopathy, skin hypopigmentation, intellectual disability, microcephaly, CMC. Ig: Decreased IgG2. Bc: Defective. Profound depletion of CD4+ cells.

Kabuki Sd. KMT2D (MLL2): AD. KDM6A: XL.

Typical facial abnormalities, cleft or high arched palate, skeletal abnormalities, short stature, intellectual disability, congenital heart defects, recurrent infections (otitis media, pneumonia) in $50 \%$ of patients. Autoimmunity may be present. Low IgA and occasionally low IgG.

Wiedemann-Steiner Sd. KMT2A (MLL): AD Respiratory infections; short stature; hypertelorism; hairy elbows; developmental delay, intellectual Wiedemann-Steiner Sd. KMT2A (MLL): AD Respiratory infection
disability. Hypogammaglobulinemia, decreased memory Bc.

Immunodeficiency, developmental delay and hypohomocysteinemia, IMDDHH*. Activating de-novo mutations in NFE2L2. AD. Recurrent respiratory

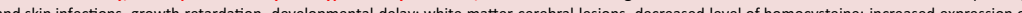
and skin infections, growth retardation, developmental delay, white matter cerebral lesson
stress response genes. Hypogammaglobulinemia. Bc: Decreased switched-mery

Tricho-Hepato-Enteric syndrome. TTC37; SKIVZL*. Respiratory infections, IUGR, wooly hair, early onset intractable diarrhea, liver cirrhosis, platelet abnormalities. Impaired IFNy production, Hypogammaglobulinemia, low antibody responses. Bc: Variably low switched-memory Bc.

Fig. 2 (continued) 
III. Predominantly Antibody deficiencies.

a: Hypogammaglobulinemia

\section{$\lg G, \lg A$ and/or $\lg M \Downarrow \downarrow$}

Exclude second causes: drugs [Hx], myeloma [bone marrow], lymphoma. Ig loss (not hypo-lgM) in urine, gastro-intestinal or skin.

$\rightarrow$ B Lymphocyte (CD19+) enumeration (CMF)

\begin{tabular}{|c|c|c|}
\hline Bc absent & \multicolumn{2}{|l|}{$B c>1 \%$} \\
\hline Severe bacterial infection. All lg isotypes & \multicolumn{2}{|c|}{ Commun Variable Immunodeficiency Phenotype } \\
\hline $\begin{array}{l}\text { X-Linked Agammaglobulinemia. BTK. } \\
\text { Some patients have detectable Ig. ProBc: NI }\end{array}$ & $\begin{array}{l}\text { CVID with no gene defect specified. Clinical phenotypes vary: most } \\
\text { have recurrent infections, some have polyclonal lymphoproliferation, } \\
\text { autoimmune cytopenias and/or granulomatous disease }\end{array}$ & $\begin{array}{l}\text { CD19 deficiency*. CD19. Recurrent infections, may } \\
\text { have glomerulonephritis. }\end{array}$ \\
\hline $\begin{array}{l}A R \text { : } \\
\mu \text { heavy chain Def. IGHM } \\
\text { Ig } \alpha \text { def* }^{*} \text {.CD79A, } \lg \beta \text { def*. CD79B }\end{array}$ & \multirow{2}{*}{$\begin{array}{l}\text { Activated p1108 syndrome (APDS) AD. Severe bacterial infections. } \\
\text { Lymphadenopathy, lymphoproliferation, lymphoma. Reduced } \\
\text { memory Bc and increased transitional Bc. PIK3CD GOF. EBV } \pm \text { CMV } \\
\text { viremia, autoimmunity. PIK3R1. Developmental delay. }\end{array}$} & $\begin{array}{l}\text { CD81 deficiency*. CD81. Recurrent infections, may } \\
\text { have glomerulonephritis. Phenocopy of CD19 } \\
\text { deficiency. }\end{array}$ \\
\hline ProBc: NI & & CD21 deficiency*. Recurrent infections. Low IgG, \\
\hline E47 transcription factor def*. TCF3 & $\begin{array}{l}\text { PTEN Deficiency (LOF)*. PTEN. AD. Lymphoproliferation, Autoimmunity. } \\
\text { Developmental delay. }\end{array}$ & \\
\hline $\begin{array}{l}\text { Severe, failure to thrive. } \\
\text { p85 def**. PIK3R1. Cytopenia. ProBc: } \downarrow \\
\text { p110ס def**. PIK3CD. Autoimmune } \\
\text { complications. }\end{array}$ & ARHGEF1 deficiency**. ARHGEF. Recurrent infections, bronchiectasis. & $\begin{array}{l}\text { TRNT1 deficiency. TRNT1. Congenital sideroblastic } \\
\text { anemia, deafness, developmental delay. B cell } \\
\text { deficiency and hypogammagl. }\end{array}$ \\
\hline ZIP7 def*. SLC39A7. Early onset & SH3KBP1 deficiency** SH3KBP1 (CIN85). XL. Severe bacterial infections. & \multirow{2}{*}{$\begin{array}{l}\text { NFKB1 deficiency. NFKB1. AD. Recurrent sinopulmonary } \\
\text { infections, COPD, EBV proliferation, autoimmune cytopenias, } \\
\text { alopecia and autoimmune thyroiditis. Ig NI or } \downarrow, \mathrm{Bc} \downarrow \text { or NI, } \\
\downarrow \text { memory Bc. }\end{array}$} \\
\hline thrombocytopenia & $\begin{array}{l}\text { SEC61A1 deficiency. }{ }^{*} \text { SEC61A1. AD, Severe recurrent respiratory tract } \\
\text { infections }\end{array}$ & \\
\hline $\begin{array}{l}\text { E47 transcription factor def*. TCF3. } \\
\text { Hoffman syndrome*. TOP2B. Facial } \\
\text { dysmorphism, limb anomalies }\end{array}$ & $\begin{array}{l}\text { RAC2 deficiency**. RAC2. AR, Recurrent sinopulmonary infections, } \\
\text { poststreptococcal glomerulonephritis; urticaria. Some have selective IgA } \\
\text { def. }\end{array}$ & $\begin{array}{l}\text { NFKB2 deficiency. NFKB2. AD. Recurrent sinopulmonary } \\
\text { infections, alopecia and endorinopathies (ie, central adrenal } \\
\text { insufficiency). Low Bc. }\end{array}$ \\
\hline \multicolumn{2}{|c|}{ CD20 deficiency**. CD20. Recurrent infections. Low IgG, $\mathrm{Nl}$ or elevated IgM and IgA. } & \multirow{2}{*}{$\begin{array}{l}\text { IKAROS haploinsufficiency. IKZF1. AD. Recurrent } \\
\text { sinopulmonary infections; increased risk of ALL, } \\
\text { autoimmunity. Decreased pro-Bc, low or normal Bc reducing } \\
\text { levels with age. }\end{array}$} \\
\hline \multicolumn{2}{|c|}{ TACI deficiency. TNFRSF13B (TACI). AD or AR. Variable clinical expression and penetrance for monoallelic variants. } & \\
\hline \multicolumn{2}{|c|}{ BAFF receptor deficiency*. TNFRSF13C (BAFF-R). Variable clinical expression. Low IgG and IgM. } & \multirow{2}{*}{$\begin{array}{l}\text { ATP6AP1 deficiency. ATP6AP1. XL. Hepatopathy, } \\
\text { leukopenia, low copper. Variable Ig findings. }\end{array}$} \\
\hline \multirow{2}{*}{\multicolumn{2}{|c|}{$\begin{array}{l}\text { TWEAK deficiency**. TWEAK (TNFSF12). AD. Pneumonia, bacterial infections, warts, thrombocytopenia. Neutropenia. Low IgM } \\
\text { and A, lack of anti-pneumococcal antibody. }\end{array}$}} & \\
\hline & & \multirow{2}{*}{$\begin{array}{l}\text { Mannosyl-oligosaccharide glucosidase deficiency } \\
\text { (MOGS)*. MOGS (GCS1). Low bacterial and viral infections } \\
\text { in comparison to the level of hypogammaglobulinemia, } \\
\text { severe neurologic disease, also known as congenital } \\
\text { disorder of glycosylation type IIb (CDG-Ilb). }\end{array}$} \\
\hline \multicolumn{2}{|c|}{$\begin{array}{l}\text { IRF2BP2 deficiency**. IRF2BP2. Recurrent infections, possible autoimmunity and inflammatory disease. Hypogammaglobulenia, } \\
\text { absent IgA. }\end{array}$} & \\
\hline
\end{tabular}

Fig. 3 Predominantly antibody deficiencies. a Hypogammaglobulinemias. b Other antibody deficiencies. AD autosomal dominant transmission, AR autosomal recessive transmission, Bc B cells, BENTA B cell expansion with NF- $\mathrm{KB}$ and $\mathrm{T}$ cell anergy, CD cluster of differentiation, CMF flow cytometry, COPD chronic obstructive pulmonary disease, def deficiency, EBV Epstein-Barr virus, GOF gain-of-function, $\mathrm{Hx}$ patient history, Ig immunoglobulins, N1 normal, XL X-linked transmission 


\section{Predominantly Antibody deficiencies.}

\section{b: Other Antibody deficiencies}

Severe Reduction in Serum IgG and IgA with Normal or elevated IgM

and Normal Numbers of $\mathrm{Bc}$ :

\section{Hyper IgM Syndromes}

AID deficiency.

AICDA. AR or AD.

Bacterial infections, enlarged lymph nodes and germinal centers. NI memory Bc, but lacking somatic

hypermutation in AR form.

UNG deficiency. UNG.

Enlarged lymph nodes and germinal centers.

INO80 def*. INO80.

Severe bacterial infections.

\section{MSH6*. MSH6.}

Family or personal history of cancer. Variable IgG,

defects, increased IgM in some, NI Bc, low switched

memory Bc.

Fig. 3 (continued)

\begin{tabular}{|c|c|}
\hline $\begin{array}{l}\text { Isotype, Light Chain, or Functional Deficiencies } \\
\text { with Generally NI Numbers of Bc }\end{array}$ & $\begin{array}{l}\text { High Bc numbers due to } \\
\text { constitutive NF-KB activation }\end{array}$ \\
\hline $\begin{array}{l}\text { Selective IgA deficiency. Unknown. } \\
\text { May be asymptomatic. Bacterial infections, autoimmunity } \\
\text { mildly increased. Very low to absent IgA with other isotypes } \\
\text { normal, normal subclasses and specific antibodies. }\end{array}$ & $\begin{array}{l}\text { CARD11 GOF. } \\
\text { CARD11. AD. BENTA syndrome }\end{array}$ \\
\hline $\begin{array}{l}\text { Transient hypogammaglobuliemia of infancy. Unknown. } \\
\text { Usually not associated with significant infections, normal ability } \\
\text { to produce antibodies to vaccine antigens. IgG and IgA decreased. }\end{array}$ & $\begin{array}{l}\text { Splenomegaly, } \\
\text { lymphadenopathy, }\end{array}$ \\
\hline $\begin{array}{l}\text { IgG subclass deficiency with IgA deficiency. Unknown. } \\
\text { Recurrent bacterial infections. May be asymptomatic. Reduced }\end{array}$ & poor vaccine responses. \\
\hline
\end{tabular}

IgA with decrease in one or more IgG subclass.

Isolated IgG subclass deficiency. Unknown.

Usually asymptomatic, a minority may have poor antibody response to specific antigens and recurrent viral/bacterial infections. Reduction in one or more IgG subclass.

Specific antibody deficiency with normal Ig levels and normal B cells. Unknown.

Reduced ability to produce antibodies to specific antigens. Ig: NI.

Ig heavy chain mutations and deletions.

Mutation or chromosomal deletion at $14 q 32$.

May be asymptomatic. One or more IgG and/or IgA subclasses as well as IgE may be absent.

Kappa chain deficiency*. IGKC.

Asymptomatic. All immunoglobulins have lambda light chain.

Selective IgM deficiency. Unknown. Pneumococcal / bacterial infections. Absent serum IgM. 


\section{Diseases of immune dysregulation. a: Hemophagocytic Lymphohistiocytosis HLH \& EBV susceptibility}

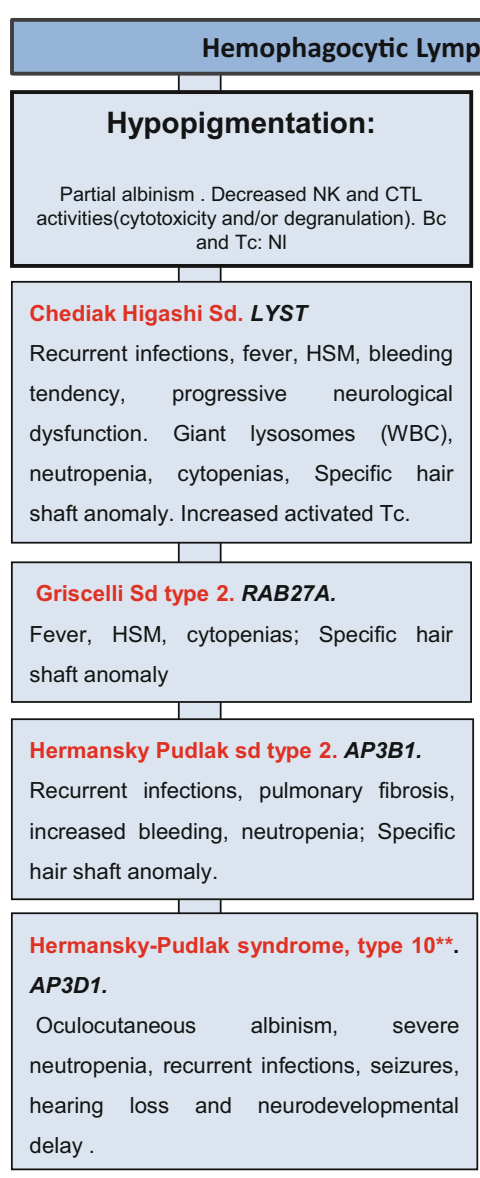

Fig. 4 Diseases of immune dysregulation. a Hemophagocytic lymphohistiocytosis. b Other diseases of immune dysregulation. Ab antibody, AD autosomal dominant transmission, Ag antigen, AIHA autoimmune hemolytic anemia, ALPS autoimmune lymphoproliferative syndrome, APS autoimmune polyendocrinopathy syndrome, AR autosomal recessive transmission, Bc B cells, CD cluster of differentiation, CMF flow cytometry, CTL cytotoxicT lymphocytes, def deficiency, DNT double negative T cells, EBV Epstein-Barr virus, FHL UNC13D. STXBP2. Enteropathy cell function.

\section{SLC7A7 deficiency. SLC7A7.}

Lysinuric protein intolerance, bleeding tendency, alveolar proteinosis HyperTc and NK cell function

\section{Susceptibility to EBV}

RASGRP1 deficiency*. RASGRP1. Recurrent pneumonia, herpes virus infections, EBV associated lymphoma. Decreased NK cell function; high IgA. BC and Tc: Poor activation, proliferation, motility

CD70 deficiency*. CD70 (TNFSF7). Hodgkin lymphoma, autoimmunity in some patients. Reduced IgM, IgG, IgA $(75 \%)$ and reduced $\mathrm{Ag}$-specific $\mathrm{Ab}$ responses $(50 \%)$. Bc:poor antibody and memory responses. Tc:low Treg, poor activation and function

UNC13D / Munc13-4 deficiency (FHL3).

Syntaxin 11 deficiency (FHL4). STX11. STXBP2 / Munc18-2 deficiency (FHL5)

FAAP24 deficiency**. FAAP24. EBV-driven lymphoproliferative disease. Increased activated Tc. Failure to kill autologous EBV transformed Bc. NI NK

inflammatory response of macrophages. NI

XL magnesium EBV and neoplasia (XMEN)*. MAGT1.XL. EBV infection, lymphoma, viral infections, respiratory and GI infections. Glycosylation disorder. Some patients can present with

neurological manifestations. Low CD4 Low recent thymic emigrant cells, poor proliferation to CD3. High B cells, high DN T cells.

PRKCD deficiency*. PRKCD. Recurrent infections, EBV chronic infection, lymphoproliferation, Recurrent/chronic bacterial and viral infections (EBV, VZV), EBV lymphoproliferation, B cell non-Hodgkin lymphoma. Tc: poor proliferation to Ag

CD137 deficiency*. TNFRSF9. EBV

lymphoproliferation, B cell lymphoma, chronic active EBV infection. Low IgA and IgG, poor response to antigens, decreased T cell proliferation

RLTPR (CARMIL2) deficiency. RLTPR. Recurrent bacterial, fungal and mycobacterial infections, viral warts, molluscum and EBV lymphoproliferative and other malignancy, atopy. Ig NI to $\downarrow$, poor T dependent antibody response. NI Bc. Tc: $\downarrow$ Treg, high CD4, poor function. familial hemophagocytic lymphohistiocytosis, GOF gain-of-function, HLH hemophagocytic lymphohistiocytosis, (H)SM (hepato)splenomegalia, IBD inflammatory bowel disease, Ig immunoglobulin, IL-10 interleukin-10, LOF loss-of-function, iNKT invariant NKT cells, NK natural killer cells, Nl normal, sd syndrome, SLE systemic lupus erythematous disease, Tc T cells, TCR T cell receptor, XL X-linked transmission
EBV associated HLH

XL, XLP1, SH2DIA.

Clinical and immunologic features triggered by EBV infection:

lymphoproliferation, Aplastic anemia, Lymphoma.

Hypogammaglobulinemia, Absent iNKT cells. Impaired NK cell and CTL cytotoxic activity Reduced Memory B cells .

SAP deficiency (FCM).

\section{XL, XLP2. XIAP.}

Splenomegaly, lymphoproliferation, Colitis, IBD, hepatitis.

Hypogammaglobulinemia, Low iNKT cells. Increased T cells susceptibility to apoptosis to CD95 and enhanced activationinduced cell death (AICD) Normal NK and CTL cytotoxic activity. XIAP def (FCM)

\section{AR, CD27 deficiency .} CD27 (TNFRSF7)

Features triggered by EBV infection, aplastic anemia,

low iNKTc lymphoma. Low Ig 


\section{Diseases of immune dysregulation. b: Syndromes with Autoimmunity and Others}

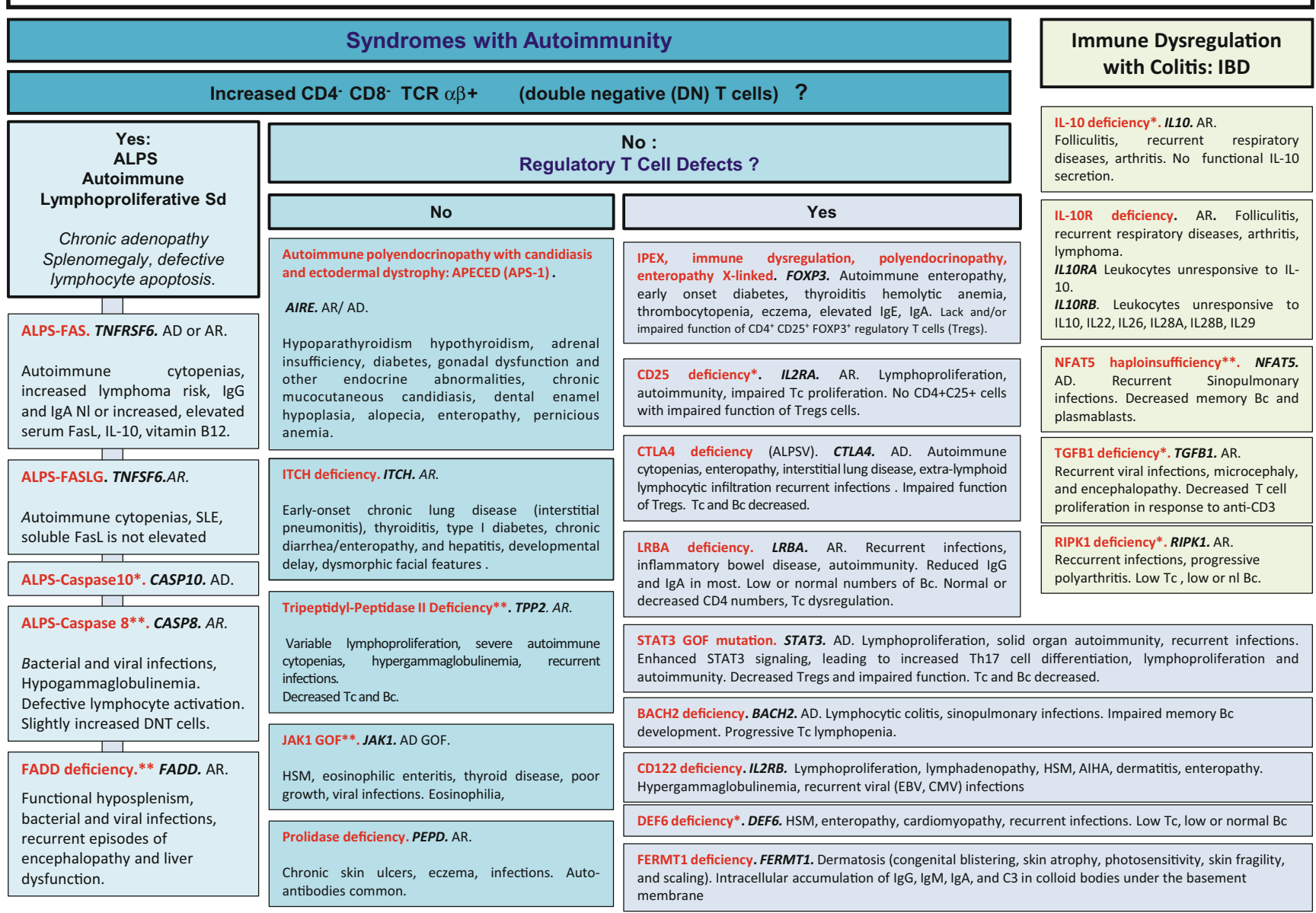

Fig. 4 (continued) 


\begin{tabular}{|c|}
\hline V. Congenital defects of phagocyte number, function, or both. \\
\hline Syndrome associated \\
\hline $\begin{array}{l}\text { Shwachman-Diamond Syndrome. DNAJC21. AR. EFL1*. AR. } \\
\text { Pancytopenia, exocrine pancreatic insufficiency. SBDS. AR. +chondrodysplasia } \\
\text { SRP54 deficiency*. SRP54. AD. Neutropenia and exocrine pancreatic insufficiency. }\end{array}$ \\
\hline $\begin{array}{l}\text { G6PC3 deficiency (SCN4). G6PC3. AR. Structural heart defects, urogenital abnormalities, inner ear } \\
\text { deafness, and venous angiectasias of trunks and limbs. Affected fonctions: Myeloid differentiation, } \\
\text { chemotaxis, } \mathrm{O}_{2} \text { - production. }\end{array}$ \\
\hline $\begin{array}{l}\text { Glycogen storage disease type } 1 \mathrm{~b} . \text { G6PT1. AR. } \\
\text { Fasting hypoglycemia, lactic acidosis, hyperlipidemia, hepatomegaly. }\end{array}$ \\
\hline Cohen syndrome. COH1. AR. Dysmorphism, mental retardation, obesity, deafness. \\
\hline $\begin{array}{l}\text { 3-Methylglutaconic aciduria. CLPB. AR. Neurocognitive developmental aberrations, microcephaly, } \\
\text { hypoglycemia, hypotonia, ataxia, seizures, cataracts, IUGR. }\end{array}$ \\
\hline $\begin{array}{l}\text { Barth Syndrome (3-Methylglutaconic aciduria type II). TAZ. XL. } \\
\text { Cardiomyopathy, myopathy, growth retardation. }\end{array}$ \\
\hline $\begin{array}{llllll}\text { Clericuzio syndrome } \quad \text { (Poikiloderma } & \text { with neutropenia). } & \text { C16ORF57 } & \text { (USB1). } & \text { AR. } \\
\text { Retinopathy, developmental delay, facial dysmorphism, poikiloderma. } & & & \end{array}$ \\
\hline $\begin{array}{l}\text { VPS45 deficiency (SCN5). VPS45. AR. } \\
\text { Extramedullary hematopoiesis, bone marrow fibrosis, nephromegaly. }\end{array}$ \\
\hline JAGN1 deficiency. JAGN1. AR. Osteopenia. Myeloid maturation arrest. \\
\hline $\begin{array}{l}\text { WDR1 deficiency. WDR1. AR. } \\
\text { Poor wound healing, severe stomatitis, neutrophil nuclei herniate. Mild neutropenia. }\end{array}$ \\
\hline $\begin{array}{l}\text { SMARCD2 deficiency*. SMARCD2. AR. } \\
\text { Developmental aberrations, bones defect, myelodysplasia }\end{array}$ \\
\hline Specific granule deficiency*. CEBPE. AR. Neutrophils with bilobed nuclei. Chronic neutropenia. \\
\hline HYOU1 deficiency**. HYOU1. AR. Hypoglycemia, inflammatory complications. \\
\hline $\begin{array}{l}\text { P14/LAMTOR2 deficiency**. LAMTOR2. AR. } \\
\text { Partial albinism, growth failure. Hypogammaglobulinemia, reduced CD8 cytotoxicity. }\end{array}$ \\
\hline
\end{tabular}

a : Neutropenia (without anti-PMN)

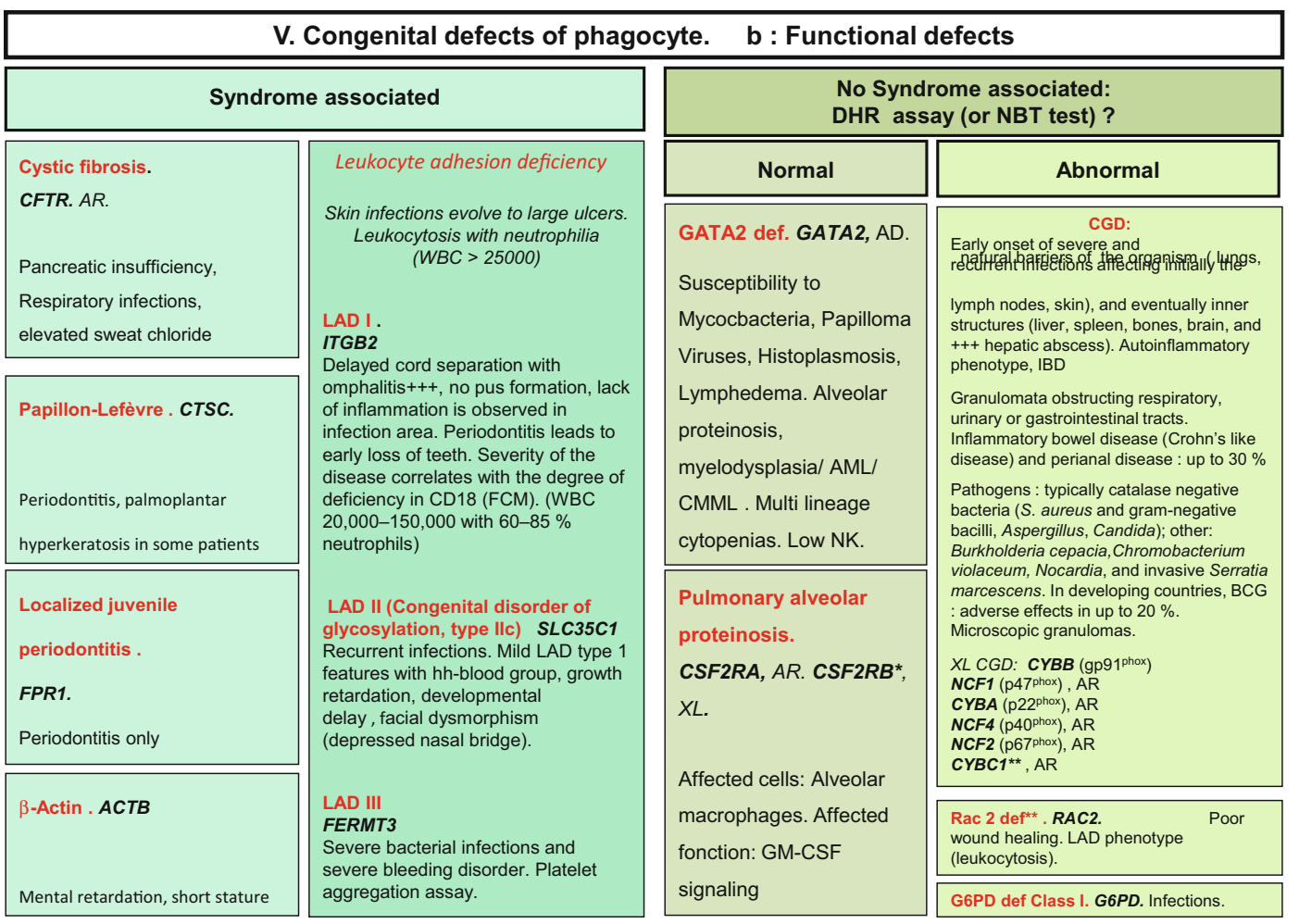

Fig. 5 Congenital defects of phagocyte number, function, or both. a Neutropenia. b Functional defects of phagocytes. AD autosomal dominant transmission, AML acute myeloid leukemia, AR autosomal recessive transmission, BCG bacillus Calmette-Guerin, $\mathrm{CD}$ cluster of differentiation, CGD chronic granulomatous disease, CMF flow cytometry, CMML chronic myelomonocytic leukemia, def deficiency,

DHR dihydrorhodamine-1,2,3, GM-CSF granulocytes/monocytes colony stimulation factor, GOF gain-of-function, IBD inflammatory bowel disease, IUGR intrauterine growth retardation, LAD leukocyte adhesion deficiency, MDS myelodysplasia, NBT nitroblue of tetrazolium, NK natural killer cells, WBC white blood cells, XL: Xlinked transmission

X-linked neutropenia/ myelodysplasia WAS GOF. WAS. XL GOF.

Myeloid maturation arrest, monocytopenia, variable lymphoid anomalies.

G-CSF receptor deficiency*. CSF3R. AR. Stress granulopoiesis disturbed

Neutropenia with combined immune deficiency *. MKL1. AR.

Mild thrombocytopenia. Lymphopenia. 
VI. Defects in Intrinsic and Innate immunity. a : Bacterial and Parasitic Infections :

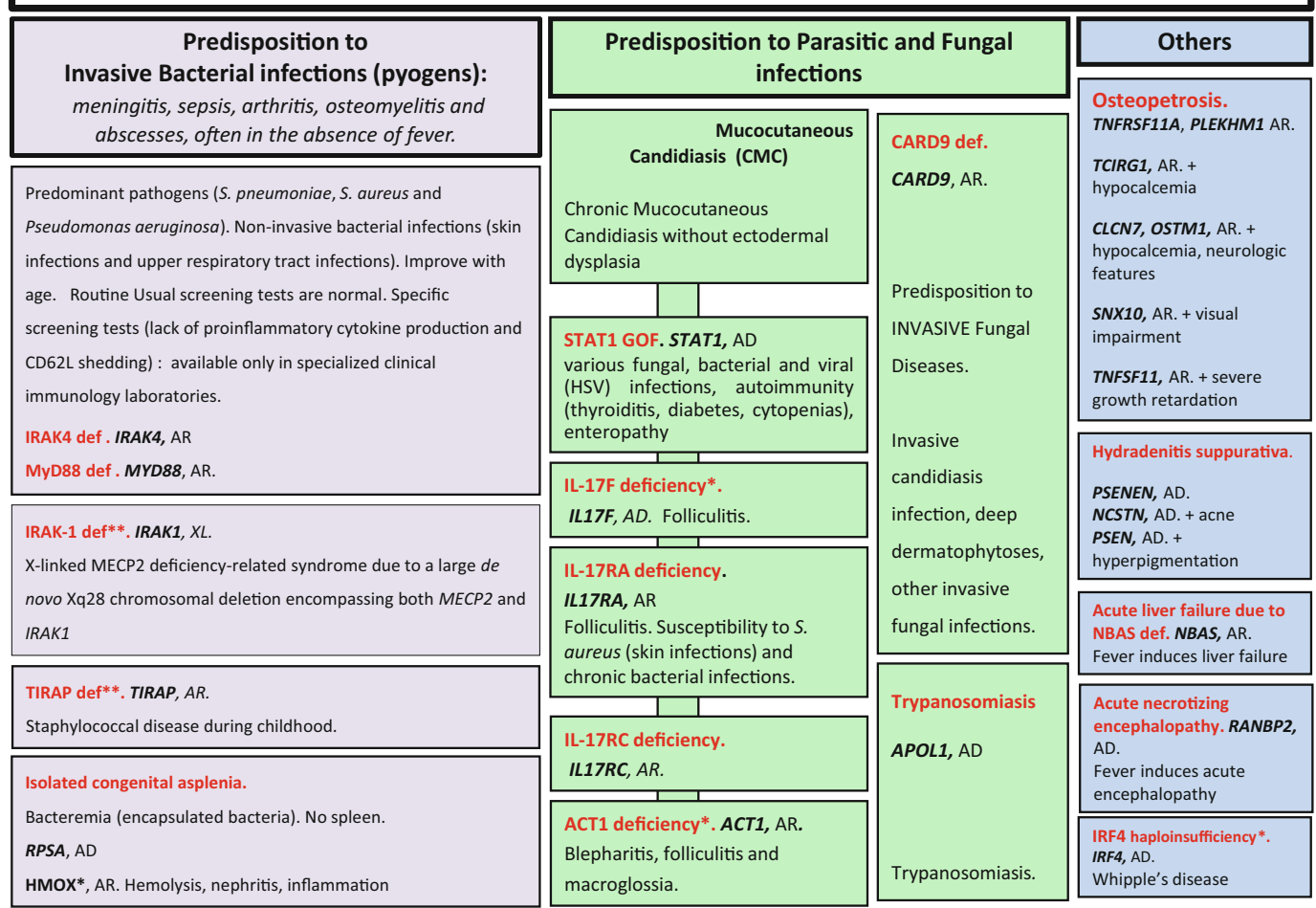

VI. Defects in Intrinsic and Innate immunity. b : MSMD and Viral infection

\begin{tabular}{|c|c|}
\hline \multicolumn{2}{|c|}{ Mendelian Susceptibility to mycobacterial disease (MSMD) } \\
\hline Severe phenotypes. & Moderate phenotypes. \\
\hline $\begin{array}{l}\text { Serious disseminated } \\
\text { BCG and environmental } \\
\text { mycobacterial infections } \\
\text { (soft tissue, bone }\end{array}$ & $\begin{array}{l}\text { With Susceptibility to Salmonella } \\
\text { IL-12 and IL-23 receptor b1 chain deficiency. } \\
\text { IL12RB1.AR. } \\
\text { IL-12p40 (IL-12 and IL-23) def. IL12B.AR. } \\
\text { IL-12Rb2 deficienc***. IL12RB2. AR } \\
\text { IL-23R deficiency**. IL23R. AR. } \\
\text { STAT1 LOF STAT1(AD) } \\
\text { Partial IFNYR1, IFNGR1. AR. } \\
\text { Partial IFNYR2, IFNGR2.AR. } \\
\text { AD IFNGR1 IFNGR1. AD. Mycobacterial } \\
\text { osteomyelitis } \\
\text { SPPL2a deficiency*. SPPL2A. AR. } \\
\text { Tyk2 deficiency, TYK2. AR. } \\
\text { Susceptibility to viruses, +/- elevated IgE. multiple } \\
\text { cytokine signaling defect. P1104A TYK2 } \\
\text { homozygosity MSMD or tuberculosis. }\end{array}$ \\
\hline marrow, lungs, skin, & $\begin{array}{l}\text { Macrophage gp91 phox deficiency CYBB, XL } \\
\text { IRF8 deficiency, IRF8 AD }\end{array}$ \\
\hline bones and lymph nodes), & $\begin{array}{l}\text { ISG15 Def, ISG15. AR. Brain calcification. } \\
\text { IFNg production defect. }\end{array}$ \\
\hline Salmonella spp., Listeria & $\begin{array}{l}\text { IRF8 deficiency, IRF8 AR Multiple other infectious } \\
\text { agents. Myeloproliferation }\end{array}$ \\
\hline monocytogenes and & $\begin{array}{l}\text { RORyt deficiency*. RORC AR. Susceptibility to } \\
\text { Candida. IFNg productiondefect, } \\
\text { complete absence of IL-17A/F-producing TC }\end{array}$ \\
\hline viruses & $\begin{array}{l}\text { JAK1 (LOF)*, JAK1. AR. Susceptibility to viruses, } \\
\text { urothelial carcinoma. } \downarrow \text { IFNg production. }\end{array}$ \\
\hline
\end{tabular}

Fig. 6 Defects in intrinsic and innate immunity. a Bacterial and parasitic infections. b MSMD and viral infection. AD autosomal dominant transmission, AR autosomal recessive transmission, BCG bacillus Calmette-Guerin, CD cluster of differentiation, CMC chronic mucocutaneous candidiasis, EBV Epstein-Barr virus, GOF gain-offunction, IFNg interferon gamma, HHV6 human herpes virus type 6,

\begin{tabular}{|c|c|c|}
\hline \multicolumn{3}{|c|}{ Predominant susceptibility to viral infection } \\
\hline $\begin{array}{l}\text { Epidermodysplasia } \\
\text { verruciformis (HPV) }\end{array}$ & $\begin{array}{l}\text { Predisposition to } \\
\text { Severe Viral Infection }\end{array}$ & $\begin{array}{l}\text { Herpes simplex } \\
\text { Encephalitis }\end{array}$ \\
\hline \multirow{4}{*}{$\begin{array}{l}\text { HPV (group B1) } \\
\text { infections and cancer of } \\
\text { the skin } \\
\text { EVER1 def. TMC6.AR. } \\
\text { EVER2 def. TMC8. AR. } \\
\text { CIB1 def. CIB1. AR. }\end{array}$} & $\begin{array}{l}\text { STAT1 Def (AR LOF). STAT1. } \\
\text { (+ Mycobacteria) }\end{array}$ & \multirow{3}{*}{\begin{tabular}{|l} 
Dominant clinical \\
phenotype is Herpes \\
simplex encephalitis (HSE) \\
during primary infection \\
with herpes simplex virus \\
type 1 (HSV1), usually \\
between 3 months and 6 \\
years of age. Incomplete \\
clinical penetrance for all \\
etiologies listed here.
\end{tabular}} \\
\hline & $\begin{array}{l}\text { STAT2 deficiency*. STAT2. } \\
\text { AR. Disseminated vaccine- } \\
\text { strain measles }\end{array}$ & \\
\hline & $\begin{array}{l}\text { IRF7 deficiency**. IRF7. AR. } \\
\text { IRF9 deficiency*. IRF9. AR. } \\
\text { Severe influenza disease. }\end{array}$ & \\
\hline & $\begin{array}{l}\text { IFNAR1 deficiency*. IFNAR1 } \\
\text { AR. Severe disease caused by } \\
\text { Yellow Fever vaccine and }\end{array}$ & $\begin{array}{l}\text { Routine screening tests } \\
\text { are normal. }\end{array}$ \\
\hline WHIM (Warts, & & \multirow{3}{*}{$\begin{array}{l}\text { Specific tests examining } \\
\text { the TLR3 pathway: } \\
\text { marked decrease in the } \\
\text { ability of patient's } \\
\text { fibroblasts to produce IFN } \\
\alpha \text { and } \beta \text { in response to } \\
\text { HSV1 infection. }\end{array}$} \\
\hline Hypogammaglobuline & $\begin{array}{l}\text { IFNAR2 deficiency**. } \\
\text { IFNAR2 AR. Disseminated } \\
\text { vaccine-strain measles, } \\
\text { HHV6. No response to IFN- } \alpha \text {. }\end{array}$ & \\
\hline \multirow{2}{*}{$\begin{array}{l}\text { mia, infections, myelo- } \\
\text { kathexis) sd } \\
\text { CXCR4 AD GOF. }\end{array}$} & $\begin{array}{l}\text { CD16 deficiency*. FCGR3A. } \\
\text { AR. Severe herpes viral } \\
\text { infections, particularly VZV, }\end{array}$ & \\
\hline & EBV, and HPV. & \multirow{2}{*}{$\begin{array}{l}\text { UNC93B1 (AR), TRAF3** } \\
\text { (AD), TICAM1 (TRIF)* } \\
\text { (AR,AD), TBK1* (AD), } \\
\text { IRF3* (AD) }\end{array}$} \\
\hline \multirow{3}{*}{$\begin{array}{l}\text { Warts (HPV) infection, } \\
\text { neutropenia, low B cell } \\
\text { number, hypogamma- } \\
\text { globulinemia. }\end{array}$} & $\begin{array}{l}\text { MDA5 deficiency (LOF)*. } \\
\text { IFIH1. AR. Rhinovirus and } \\
\text { other RNA viruses }\end{array}$ & \\
\hline & $\begin{array}{l}\text { RNA poymerase III def*. } \\
\text { POLR3A. POLR3C. POLR3F. } \\
\text { AD. Severe VZV infection. }\end{array}$ & \multirow{2}{*}{$\begin{array}{l}\text { TLR3 }(A D, A R),+ \text { severe } \\
\text { pulmonary influenza, VZV } \\
\text { DBR1* (AR) +other viral } \\
\text { infections of the } \\
\text { brainstem }\end{array}$} \\
\hline & $\begin{array}{l}\text { IL-18BP def**. IL18BP. AR. } \\
\text { Fulminant viral hepatitis }\end{array}$ & \\
\hline
\end{tabular}

HPV human papillomavirus, HSV herpes simplex virus, LOF loss-offunction, MSMD Mendelian susceptibility to mycobacterial disease, NK natural killer cells, RNA ribonucleic acid, sd syndrome, Tc T cells, TLR3 Toll-like receptor type 3, VZV varicella zoster virus, XL X-linked transmission 


\begin{tabular}{|c|c|c|}
\hline \multicolumn{3}{|c|}{ VIla. Auto-inflammatory disorders } \\
\hline \multirow{2}{*}{$\begin{array}{c}\text { Recurrent inflammation } \\
\text { Recurrent fever }\end{array}$} & Systemic inflammation with urticaria rash & Others \\
\hline & \multirow{2}{*}{$\begin{array}{l}\text { Familial Cold Autoinflammatory Syndrome (CAPS) *. } \\
\text { NLRP3, NLRP12. AD GOF DA: } 24-48 \mathrm{H} \\
\text { Non-pruritic urticaria, arthritis, chills, fever and } \\
\text { leukocytosis after cold exposure. }\end{array}$} & \multirow{3}{*}{$\begin{array}{l}\text { CANDLE sd (chronic atypical neutrophilic } \\
\text { dermatitis with lipodystrophy). } \\
\text { PSMB8, AR and AD. Contractures, } \\
\text { panniculitis, ICC, fevers. } \\
\text { PSMG2, AR. Panniculitis, lipodystrophy, } \\
\text { AIHA. } \\
\text { (Variants in PSMB4, PSMBg, PSMA3, and POMP have } \\
\text { been proposed to cause a similar CANDLE phenotype in } \\
\text { compound heterozygous monogenic, digenic, and AD } \\
\text { monogenic models). }\end{array}$} \\
\hline $\begin{array}{l}\text { Familial Mediterranean Fever (FMF) * } \\
\text { MEFV. AR or AD (Usually M694del variant) }\end{array}$ & & \\
\hline $\begin{array}{l}\text { DA: 1-4 days FA : Variable. } \\
\text { Polyserositis, Abdominal pain, Arthritis, } \\
\text { Amyloidosis. Erysipelas-like erythema. }\end{array}$ & \multirow{2}{*}{$\begin{array}{l}\text { Muckle Wells syndrome (CAPS) * NLRP3. AD GOF. } \\
\text { Ethnic group : North European } \\
\text { Continuous fever. Often worse in the evenings. } \\
\text { Urticaria, Deafness (SNHL), Conjunctivitis, Amyloidosis. }\end{array}$} & \\
\hline $\begin{array}{l}\text { Predisposes to vasculitis and inflammatory } \\
\text { bowel disease. }\end{array}$ & & \multirow{2}{*}{$\begin{array}{l}\text { COPA defect. COPA. AD } \\
\text { Autoimmune inflammatory arthritis and } \\
\text { interstitial lung disease with Th17 } \\
\text { dysregulation and autoantibody production }\end{array}$} \\
\hline Colchicine-responsive +++. & \multirow{2}{*}{$\begin{array}{l}\text { Neonatal onset multisystem inflammatory disease } \\
\text { (NOMID) or chronic infantile neurologic cutaneous and } \\
\text { articular syndrome (CINCA) *. NLRP3. AD GOF. } \\
\text { Neonatal onset rash, with continuous fever and } \\
\text { inflammation. Aseptic and chronic meningitis, chronic } \\
\text { arthropathy. Mental retardation, Sensorineural deafness. } \\
\text { and Visual loss in some patients. }\end{array}$} & \\
\hline $\begin{array}{l}\text { DA: } 3-7 \text { days FA: } 1-2 \text { monthly. } \\
\text { Cervical adenopathy. Oral aphtosis. }\end{array}$ & & $\begin{array}{l}\text { NLRC4-MAS (macrophage activating } \\
\text { syndrome)*. NLRC4. } \\
\text { AD GOF. Severe enterocolitis and } \\
\text { macrophage activation syndrome (HLH). } \\
\text { Triggered by cold exposure. }\end{array}$ \\
\hline $\begin{array}{l}\text { Cervical adenopathy. Oral aphtosis. } \\
\text { Diarrhea. Mevalonate aciduria during } \\
\text { attacks. Leukocytosis with high IgD levels. }\end{array}$ & \multirow{2}{*}{$\begin{array}{l}\text { A20 haploinsufficiency TNFAIP3. AD LOF. Arthralgia, } \\
\text { mucosal ulcers, ocular inflammation. }\end{array}$} & $\begin{array}{l}\text { NLRP1 GOF. NLRP1. AD GOF. } \\
\text { Palmoplantar carcinoma, corneal scarring; }\end{array}$ \\
\hline \multirow{4}{*}{$\begin{array}{l}\text { TNF receptor-associated periodic } \\
\text { syndrome; TRAPS. TNFRSF1A. AD. } \\
\text { DA: 1-4 weeks FA: Variable } \\
\text { Prolonged fever. Serositis, rash, Periorbital } \\
\text { edema and conjunctivitis. }\end{array}$} & & $\begin{array}{l}\text { recurrent respiratory papillomatosis. } \\
\text { Increased IL1 } \beta \text {. }\end{array}$ \\
\hline & \multirow{2}{*}{$\begin{array}{l}\text { PLAID (PLCg2 associated antibody deficiency and } \\
\text { immune dysregulation), or APLAID*. PLC2G. AD GOF. } \\
\text { Cold Urticaria. Impaired humoral immunity. } \\
\text { Hypogammaglobulinemia, autoinflammation. }\end{array}$} & $\begin{array}{l}\text { ALPI deficiency*. ALP1. AR. } \\
\text { TRIM22 def*. TRIM22. AR } \\
\text { Inflammatory bowel disease. }\end{array}$ \\
\hline & & \multirow{2}{*}{$\begin{array}{l}\text { T-cell lymphoma subcutaneous } \\
\text { panniculitis-like (TIM3 deficiency). HAVCR2. } \\
\text { AR. Panniculitis, HLH, polyclonal cutaneous } \\
\text { T cell infiltrates or T-cell lymphoma }\end{array}$} \\
\hline & $\begin{array}{l}\text { NLRP1 deficiency*. NLRP1. AR. } \\
\text { Dyskeratosis, autoimmunity and arthritis. }\end{array}$ & \\
\hline
\end{tabular}

\begin{tabular}{|c|c|c|}
\hline \multicolumn{3}{|c|}{ VIIb. Auto-inflammatory diso } \\
\hline \multicolumn{2}{|c|}{ Sterile inflammation ( skin / bone / joints ) } & Type 1 Interferonopathies \\
\hline Predominant on the bone / joints & Predominant on the skin & \multirow{2}{*}{$\begin{array}{l}\text { Progressive encephalopathy, ICC, Cerebral atrophy, HSMG, } \\
\text { leukodystrophy ' Thrombocytopenia, Elevated hepatic } \\
\text { transaminases. Chronic cerebrospinal fluid (CSF) lymphocytosis } \\
\text { Aicardi-Goutieres Syndromes: } \\
\text { TREX1 AR-AD (+SLE, FCL), RNASEH2A, RNASEH2B (+SP), } \\
\text { RNASEH2C, SAMHD1 (+ FCL), ADAR1 (+BSN, SP), IFIH1 GOF AD (+ } \\
\text { SLE, SP, SMS), DNASE2 }\end{array}$} \\
\hline $\begin{array}{l}\text { Pyogenic sterile arthritis, pyoderma } \\
\text { gangrenosum, acne (PAPA) syndrome, } \\
\text { hyperzincemia and hyper- } \\
\text { calprotectinemia. PSTPIP1 (C2BP1). AD }\end{array}$ & $\begin{array}{l}\text { Blau syndrome. NOD2 (CARD15). AD. } \\
\text { Continuous inflammation. } \\
\text { Uveitis, Granulomatous synovitis, Camptodactyly, } \\
\text { Rash, Cranial neuropathies, 30\% develop Crohn }\end{array}$ & \\
\hline DA: 5 days FA: Fixed interval : & & \\
\hline $\begin{array}{lll}\text { Destructive } & \text { arthritis, } & \text { Pyoderma }\end{array}$ & CAMPS CARD14. AD. Psoriasis. & \multirow{2}{*}{$\begin{array}{l}\text { Spondyloenchondro-dysplasia with immune dysregulation } \\
\text { (SPENCDI). ACP5. } \\
\text { Short stature, SP, ICC, SLE-like auto-immunity (Sjögren's } \\
\text { syndrome, hypothyroidism, inflammatory myositis, Raynaud's s. } \\
\text { disease and vitiligo), hemolytic anemia, thrombocytopena, } \\
\text { skeletal dysplasia, possibly recurrent bacterial and viral infections. }\end{array}$} \\
\hline $\begin{array}{l}\text { gangrenosum, inflammatory skin rash, } \\
\text { Myositis. Acute-phase response during } \\
\text { attacks }\end{array}$ & \multirow{2}{*}{$\begin{array}{l}\text { DITRA. (Deficiency of IL-36 receptor antagonist). } \\
\text { IL-36RN. AR. } \\
\text { Life-threatening, multisystemic inflammatory } \\
\text { disease characterized by episodic widespread, } \\
\text { pustular psoriasis, malaise, and leukocytosis. }\end{array}$} & \\
\hline $\begin{array}{l}\text { Chronic recurrent multifocal osteomyelitis } \\
\text { and congenital dyserythropoieticanemia } \\
\text { (Majeed syndrome). LPIN2. AR }\end{array}$ & & $\begin{array}{l}\text { STING-associated vasculopathy, infantile-onset. TMEM173. } \\
\text { Early-onset inflammatory disease, Skin vasculopathy, inflammatory } \\
\text { lung disease, systemic autoinflammation and ICC, FCL. }\end{array}$ \\
\hline \multirow{2}{*}{$\begin{array}{l}\text { DA : Few days FA : 1-3 / month } \\
\text { Chronic recurrent multifocal osteomyelitis, } \\
\text { severe pain, tender soft tissue swelling, } \\
\text { Transfusion-dependent anemia, cutaneous } \\
\text { inflammatory disorders }\end{array}$} & $\begin{array}{l}\text { Early onset diarrhea and skin lesions. Severe } \\
\text { bacteremia. } \\
\text { Defective TNF } \alpha \text { production. }\end{array}$ & $\begin{array}{l}\text { ADA2 deficiency. CECR1. Polyarteritis nodosa, childhood-onset, } \\
\text { early-onset recurrent ischemic stroke and fever, Livedo racemosa, } \\
\text { some patients develop hypogammaglobulinemia }\end{array}$ \\
\hline & \multirow{3}{*}{$\begin{array}{l}\text { SLC29A3 mutation. SLC29A3 . AR. } \\
\text { Hyperpigmentation hypertrichosis, histiocytosis- } \\
\text { lymphadenopathy plus syndrome }\end{array}$} & \multirow{2}{*}{$\begin{array}{l}\text { XL reticulate pigmentary disorder. POLA1. Hyper- } \\
\text { pigmentation, reticulate pattern. Inflammatory lung and } \\
\text { Gastroenteritis or colitis. Corneal scarring, characteristicfacies }\end{array}$} \\
\hline \multirow{3}{*}{$\begin{array}{l}\text { DIRA (Deficiency of the Interleukin } 1 \\
\text { Receptor Antagonist) IL1RN. AR } \\
\text { Continuous inflammation. } \\
\text { Neonatal onset of sterile multifocal } \\
\text { osteomyelitis, periostitis and pustulosis. }\end{array}$} & & \\
\hline & & USP18 def *. USP18. TORCH like syndrome. \\
\hline & \multirow{2}{*}{$\begin{array}{l}\text { Otulipenia/ORAS*. OTULIN. AR. } \\
\text { Neonatal onset of recurrent fever, Arthralgia, } \\
\text { lipodystrophy. Dermatitis, diarrhea, Neutrophilia }\end{array}$} & \multirow{2}{*}{$\begin{array}{l}\text { Pediatric systemic lupus erythematosus. DNASE1L3. Very } \\
\text { early onset SLE, reduced complement levels, autoantibodies } \\
\text { (dsDNA, ANCA), lupus nephritis, hypocomplementemic } \\
\text { urticarial vasculitis syndrome. }\end{array}$} \\
\hline \multirow{2}{*}{$\begin{array}{l}\text { Cherubism. SH3BP2. } \\
\text { AR. } \\
\text { Bone degeneration in jaws }\end{array}$} & & \\
\hline & $\begin{array}{l}\text { AP1S3 deficiency*. AP1S3. AR. } \\
\text { Pustular psoriasis }\end{array}$ & $\begin{array}{l}\text { OAS1 def*. OAS1. AD GOF. Pulmonary alveolar proteinosis, } \\
\text { skin rash. }\end{array}$ \\
\hline
\end{tabular}

Fig. 7 a, b Autoinflammatory disorders. AD autosomal dominant transmission, ANCA anti-neutrophilic cytoplasmic autoantibody, AR autosomal recessive transmission, BSN bilateral striatal necrosis, CAPS cryopyrin-associated periodic syndrome, DA duration of acute inflammation episode, dsDNA double-stranded deoxyribonucleic acid, FA frequency of acute inflammation episode, FCL familial chilblain lupus, GOF gain-of-function, HLH hemophagocytic lymphohistiocytosis, HSM hepatosplenomegalia, ICC intracranial calcifications, IL interleukin, LOF loss-of-function, sd syndrome, SLE systemic lupus erythematosus, SMS Singleton-Merten syndrome, SNHL sensorineural hearing loss, SP spastic paraparesis, TORCH toxoplasmosis, other, rubella, cytomegalovirus, and herpes infections 


\section{Complement deficiencies}

\section{Susceptibility to infections}

\begin{tabular}{|c|c|}
\hline & High \\
\hline \multicolumn{2}{|c|}{$\begin{array}{c}\text { Disseminated } \\
\text { Neisserial infections }\end{array}$} \\
\hline $\begin{array}{l}\text { Absent } \text { CH50 and AH50 } \\
\text { hemolytic activity. } \\
\text { Defective bactericidal } \\
\text { activity. }\end{array}$ & $\begin{array}{l}\text { Normal CH50 } \\
\text { Absent AH50 }\end{array}$ \\
\hline C5 def. C5 & activity \\
\hline C6 def. C6 & \\
\hline $\begin{array}{l}\text { C7 def. C7. } \\
+ \text { Vasculitis }\end{array}$ & def. \\
\hline c8 def. & \\
\hline$C 8 A, C 8 B, C 8 G$ & Factor D \\
\hline C9 def. $C 9$. & def. \\
\hline Mild susceptibility. & CFD. AR. \\
\hline
\end{tabular}

\section{Recurrent pyogenic infections}

C3 LOF. C3. AR.
Absent CH50 and AH50
hemolytic activity,
defective opsonization
and humoral response

\section{Ficolin 3 def.} FCN3. AR.

Infections mainly in the lungs; abscesses, necrotizing enterocolitis in infancy; selective antibody defect to Pneumococcal polysaccharides. Absence of
complement activation complement activation
by the Ficolin 3 pathway

Factor B. CFB LOF.

AR. Infections with encapsulated

organisms. Deficient activation of the alternative pathway

\begin{tabular}{|c|c|c|}
\hline \multicolumn{3}{|c|}{ Low } \\
\hline $\begin{array}{l}\text { SLE-like syndrome. } \\
\text { Infections with encapsulated }\end{array}$ & $\begin{array}{l}\text { Atypical Hemolytic } \\
\text { Uremic Syndrome }\end{array}$ & Others \\
\hline Absent $\mathrm{CH} 50$ hemolytic activity & $\begin{array}{l}\text { C3 GOF. C3. AD. } \\
\text { Glomerulonephritis. Increased activation of } \\
\text { complement }\end{array}$ & \multirow{3}{*}{$\begin{array}{l}\text { C1inhibitor. } \\
\text { SERPING1. } \\
\text { AD, Hereditary } \\
\text { angioedema. } \\
\text { Spontaneous } \\
\text { activation of the } \\
\text { complement pathway } \\
\text { with consumption of } \\
\text { C4/C2 }\end{array}$} \\
\hline C1q def. C1QA, C1QB, C1QC. & $\begin{array}{l}\text { Factor B GOF. CFB. AD. Increased } \\
\text { spontaneous AH50 }\end{array}$ & \\
\hline $\begin{array}{l}\text { C1r def. C1R. Ehlers Danlos } \\
\text { phenotype }\end{array}$ & \multirow{2}{*}{$\begin{array}{l}\text { Factor H def. CFH. AR or AD. } \\
\text { Infections, disseminated neisserial } \\
\text { infections, preeclampsia. Spontaneous } \\
\text { activation of the alternative complement } \\
\text { pathway with consumption of C3 }\end{array}$} & \\
\hline C1s def.C1S. & & \multirow{3}{*}{$\begin{array}{l}\text { Membrane Attack } \\
\text { Complex Inhibitor } \\
\text { deficiency. } \\
\text { CD59. Hemolytic } \\
\text { anemia. } \\
\text { Polyneuropathy. }\end{array}$} \\
\hline $\begin{array}{l}\text { Multiple autoimmune diseases; } \\
\text { Ehlers Danlos phenotype }\end{array}$ & \multirow{2}{*}{$\begin{array}{l}\text { Factor } \mathrm{H} \text {-related protein } \\
\text { deficiencies. CFHR1-5. } \\
\text { AR or AD. Later onset, disseminated } \\
\text { neisserial infections. Normal CH50, AH50, } \\
\text { autoantibodies to Factor } \mathrm{H} \text {. }\end{array}$} & \\
\hline C2 def. C2. & & \\
\hline $\begin{array}{l}\text { Vasculitis, Polymyositis, } \\
\text { atherosclerosis }\end{array}$ & \multirow{2}{*}{$\begin{array}{l}\text { Factor I deficiency. AR. Infections, } \\
\text { disseminated neisserial infections, } \\
\text { preeclampsia. Spontaneous activation } \\
\text { of the alternative complement } \\
\text { pathway with consumption of C3 }\end{array}$} & \multirow{2}{*}{$\begin{array}{l}\text { CD55 deficiency } \\
\text { (CHAPLE disease). } \\
\text { CD55. AR. Protein } \\
\text { losing enteropathy, } \\
\text { thrombosis }\end{array}$} \\
\hline Complete C4 def. & & \\
\hline Partial deficiency is common & $\begin{array}{l}\text { Thrombomodulin def. THBD. AD. } \\
\text { Normal CH50, AH50 }\end{array}$ & \multirow{2}{*}{$\begin{array}{l}\text { Periodontal Ehlers } \\
\text { Danlos. C1R,C1S. } \\
\text { AD GOF. } \\
\text { Hyperpigmentation } \\
\text { skin fragility. } \\
\text { Normal CH50. }\end{array}$} \\
\hline $\begin{array}{l}\text { (either } \mathrm{C} 4 \mathrm{~A} \text { or } \mathrm{C} 4 \mathrm{~B} \text { ) and appears } \\
\text { to have a modest effect on host } \\
\text { defense }\end{array}$ & $\begin{array}{l}\text { Membrane Cofactor Protein } \\
\text { deficiency. CD46.AD,Glomerulone- } \\
\text { phritis. Infections, preeclampsia. Inhibitor } \\
\text { of complement alternate pathway, } \\
\text { decreased C3b binding }\end{array}$ & \\
\hline
\end{tabular}

Fig. 8 Complement deficiencies. AD autosomal dominant transmission, AH50 alternate pathway hemolytic activity, AR autosomal recessive transmission, CH50 complement hemolytic activity, def deficiency,
GOF gain-of-function, LOF loss-of-function, sd syndrome, SLE systemic lupus erythematosus, XL X-linked transmission 


\section{Bone marrow failure}

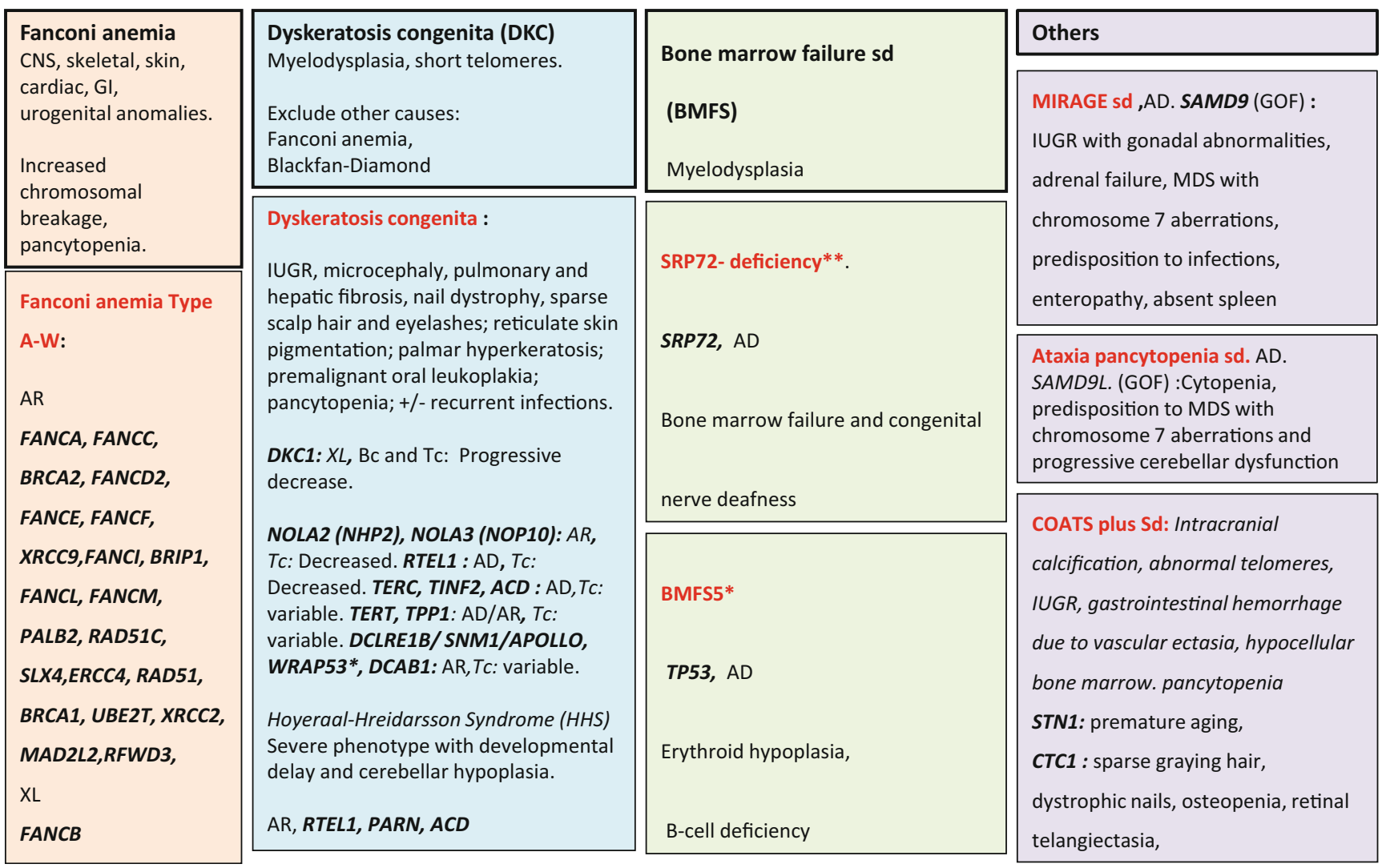

Fig. 9 Bone marrow failure disorders. AD autosomal dominant transmission, AR autosomal recessive transmission, Bc B cells, BMFS bone marrow failure syndrome, CNS central nervous system, DKC dyskeratosis congenita, GI gastrointestinal, GOF gain-of-function, IUGR intrauterine growth retardation, MDS myelodysplasia, sd syndrome, Tc T cells, XL X-linked transmission 


\section{Phenocopies of PID}

\section{Associated with}

\section{Somatic Mutations}

Splenomegaly, lymphadenopathy,

autoimmune cytopenias. Defective lymphocyte apoptosis.

\section{ALPS-SFAS}

(somatic mutations in TNFRSF6)/ ALPS-FAS

(ALPS type Im)

RALD. RAS-associated autoimmune leukoproliferative disease. (ALPS Like); N-RAS GOF, K-RAS GOF

Sporadic; granulocytosis, monocytosis/ALPS-like

Cryopyrinopathy, (Muckle-Wells /CINCA/NOMID-like

syndrome). NRLP3.

Urticaria-like rash, arthropathy, neurological symptoms

Hypereosinophilic syndrome due to somatic mutations in STAT5b. STAT5b. GOF.

Atopic dermatitis, urticarial rash, diarrhea. Eosinophilia.

\section{Associated with}

Auto-Antibodies

Chronic mucocutaneous candidiasis (isolated or with APECED syndrome) AutoAb to IL-17 and/or IL-22.

Endocrinopathy, chronic mucocutaneous candidiasis /CMC. Germline mutation in AIRE

Adult-onset immunodeficiency with susceptibility to mycobacteria. Auto-Ab to IFNg.

Mycobacterial, fungal, salmonella, VZV infections /MSMD or CID.

Recurrent skin infection. AutoAb to IL-6.

Staphylococcal infections / STAT3 deficiency

Pulmonary alveolar proteinosis . AutoAb to GM-CSF.

Pulmonary alveolar proteinosis, cryptococcal meningitis, disseminated nocardiosis/CSF2RA deficiency

Acquired angiooedema. AutoAb to $\mathbf{C} 1$ inhibitor. Angioedema /C1 inhibitor deficiency

Atypical Hemolytic Uremic Syndrome . AutoAb to Factor H. Spontaneous activation of the alternative complement pathway

Thymoma with hypogammaglobulinemia (Good syndrome). AutoAb to various cytokines. Invasive bacterial, viral or opportunistic infections, autoimmunity, PRCA, lichen planus, cytopenia, colitis, chronic diarrhea. No B cells.

Fig. 10 Phenocopies of PID. ALPS autoimmune lymphoproliferative syndrome, AutoAb autoantibodies, CID combined immunodeficiency, CMC chronic mucocutaneous candidiasis, GOF gain-of-function, MSMD Mendelian susceptibility to mycobacterial disease, PRCA pure red cell aplasia

\section{Conclusion}

This phenotypic classification of IEI forms a diagnostic resource, aimed to complement the 2019 IUIS genotypic classification. These figures serve as diagnostic orientation tools for patients with any of the typical phenotypic presentations of IEI, whether clinical or biological. They were designed for, and will hopefully be useful to physicians and biologists who are not experts in the field of IEI. We hope that these figures can help them reach a diagnosis of IEI when encountering patients whose clinical or biological phenotypes are evocative of IEI.

\section{Compliance with Ethical Standards}

Conflict of Interest The authors declare that they have no conflict of interest.

Open Access This article is licensed under a Creative Commons Attribution 4.0 International License, which permits use, sharing, adaptation, distribution and reproduction in any medium or format, as long as you give appropriate credit to the original author(s) and the source, provide a link to the Creative Commons licence, and indicate if changes were made. The images or other third party material in this article are included in the article's Creative Commons licence, unless indicated otherwise in a credit line to the material. If material is not included in the article's Creative Commons licence and your intended use is not permitted by statutory regulation or exceeds the permitted use, you will need to obtain permission directly from the copyright holder. To view a copy of this licence, visit http://creativecommons.org/licenses/by/4.0/.

\section{References}

1. Tangye SG, Al-Herz W, Bousfiha A,Chatila T, CunninghamRundles C, Etzioni A, et al. Human Inborn Errors of Immunity: 2019 Update on the Classification from the International Union of Immunological Societies Expert Committee. J Clin Immunol (2020). https://doi.org/10.1007/s10875-019-00737-x

2. Bousfiha AA, Jeddane L, Ailal F, Benhsaien I, Mahlaoui N, Casanova $\mathrm{JL}$, et al. Primary immunodeficiency diseases worldwide: more common than generally thought. J Clin Immunol. 2013;33(1):1-7.

3. Bousfiha A, Jeddane L, Picard C, Ailal F, Gaspar HB, Al-Herz W, et al. The 2017 IUIS phenotypic classification for primary immunodeficiencies. J Clin Immunol. 2018;38(1):129-43. 
4. Jeddane L, Ouair H, Benhsaien I, El Bakkouri J, Bousfiha AA. Primary immunodeficiency classification on smartphone. J Clin Immunol. 2017;37(1):1-2.

5. Shearer WT, Dunn E, Notarangelo LD, Dvorak CC, Puck JM, Logan $\mathrm{BR}$, et al. Establishing diagnostic criteria for severe combined immunodeficiency disease (SCID), leaky SCID, and Omenn syndrome: the primary immune deficiency treatment consortium experience. $\mathrm{J}$ Allergy Clin Immunol. 2014;133(4):1092-8.

Publisher's Note Springer Nature remains neutral with regard to jurisdictional claims in published maps and institutional affiliations.

\section{Affiliations}

\section{Aziz Bousfiha ${ }^{1,2} \cdot$ Leila Jeddane $^{3} \cdot$ Capucine Picard $^{4,5} \cdot$ Waleed Al-Herz $^{6} \cdot$ Fatima Ailal $^{1} \cdot$ Talal Chatila $^{7}$. Charlotte Cunningham-Rundles ${ }^{8} \cdot$ Amos Etzioni $^{9} \cdot$ Jose Luis Franco ${ }^{10} \cdot$ Steven M Holland ${ }^{11} \cdot$ Christoph Klein $^{12}$. Tomohiro Morio ${ }^{13} \cdot$ Hans D. Ochs ${ }^{14}$. Eric Oksenhendler ${ }^{15}$. Jennifer Puck ${ }^{16}$. Troy R. Torgerson ${ }^{14}$. Jean-Laurent Casanova ${ }^{17,18,19,20} \cdot$ Kathleen E. Sullivan ${ }^{21} \cdot$ Stuart G. Tangye ${ }^{22,23}$}

1 Laboratoire d'Immunologie Clinique, d'Inflammation et d'Allergy LICIA, Faculty of Medecine and Pharmacy, King Hassan II University, Casablanca, Morocco

2 Clinical Immunology Unit, Pediatric Infectiouse Disease Departmentn Children's Hospital, Ibn Rochd University Hospital, Casablanca, Morocco

3 Laboratoire national de référence, University Mohamed VI of Health Sciences, Casablanca, Morocco

4 Study Center for Primary Immunodeficiencies, Necker Hospital for Sick Children, APHP, Paris University, Paris, France

5 Laboratory of Lymphocyte Activation and Susceptibility to EBV, INSERM UMR1163, Imagine Institute, Necker Hospital for Sick Children, Paris University, Paris, France

6 Department of Pediatrics, Faculty of Medicine, Kuwait University, Kuwait City, Kuwait

7 Division of Immunology, Children's Hospital Boston, Boston, USA

8 Departments of Medicine and Pediatrics, Mount Sinai School of Medicine, New York, USA

9 Ruth's Children's Hospital-Technion, Haifa, Israel

10 Grupo de Inmunodeficiencias Primarias, Facultad de Medicina, Universidad de Antioquia UdeA, Medellin, Colombia

11 Laboratory of Clinical Immunology \& Microbiology, National Institute of Allergy and Infectious Diseases, National Institutes of Health, Bethesda, USA
12 Dr von Hauner Children's Hospital, Ludwig-MaximiliansUniversity Munich, Munich, Germany

13 Department of Pediatrics and Developmental Biology, Tokyo Medical and Dental University (TMDU), Tokyo, Japan

14 Department of Pediatrics, University of Washington and Seattle Children's Research Institute, Seattle, USA

15 Department of Clinical Immunology, Hôpital Saint-Louis, APHP, University Paris Diderot, Sorbonne Paris, Cité, Paris, France

16 Department of Pediatrics, University of California San Francisco and UCSF Benioff Children's Hospital, San Francisco, USA

17 St. Giles Laboratory of Human Genetics of Infectious Diseases, Rockefeller Branch, The Rockefeller University, New York, USA

18 Howard Hughes Medical Institute, New York, USA

19 Laboratory of Human Genetics of Infectious Diseases, Necker Branch, INSERM UMR1163, Imagine Institute, Necker Hospital for Sick Children, Paris University, Paris, France

20 Pediatric Hematology-Immunology Unit, Necker Hospital for Sick Children Assistance Publique-Hôpitaux de Paris (APHP), Paris, France

21 Division of Allergy Immunology, Department of Pediatrics, The Children's Hospital of Philadelphia, University of Pennsylvania Perelman School of Medicine, Philadelphia, USA

22 Garvan Institute of Medical Research, Darlinghurst, Australia

23 St Vincent's Clinical School, Faculty of Medicine, UNSW, Sydney, Australia 